\title{
Controlling the crystal polymorph by exploiting the time dependence of nucleation rates
}

\author{
Laurie J. Little, ${ }^{1}$ Alice A. K. King, ${ }^{1,2}$ Richard P. Sear, ${ }^{1}$, a) and Joseph L. Keddie ${ }^{1}$ \\ ${ }^{1)}$ Department of Physics, University of Surrey, Guildford, Surrey GU2 7XH, \\ United Kingdom \\ ${ }^{2)}$ Department of Physics and Astronomy, University of Sussex, Brighton, BN1 9RH, \\ United Kingdom
}

\begin{abstract}
Most substances can crystallise into two or more different crystal lattices, called polymorphs. Despite this, there are no systems in which we can quantitatively predict the probability of one competing polymorph forming, instead of the other. We address this problem using large scale (hundreds of events) studies of the competing nucleation of the alpha and gamma polymorphs of glycine. In situ Raman spectroscopy is used to identify the polymorph of each crystal. We find that the nucleation kinetics of the two polymorphs is very different. Nucleation of the alpha polymorph starts off slowly but accelerates, while nucleation of the gamma polymorph starts off fast but then slows. We exploit this difference to increase the purity with which we obtain the gamma polymorph by a factor of ten. The statistics of the nucleation of crystals is analogous to that of human mortality, and using a result from medical statistics we show that conventional nucleation data can say nothing about what, if any, are the correlations between competing nucleation processes. Thus we can show that, with data of our form, it is impossible to disentangle the competing nucleation processes. We also find that the growth rate and the shape of a crystal depends on when it nucleated. This is new evidence that nucleation and growth are linked.
\end{abstract}

Keywords: Crystallisation | Polymorphism | Crystal nucleation | Crystal growth

\section{INTRODUCTION}

Most molecules can crystallise into more than one crystal lattice, these crystal structures are called polymorphs of that molecule. A crystal's polymorph defines its properties, and so polymorph control is crucial for intended applications. For pharmaceuticals, polymorph control is also required by regulators; without it a crystalline drug cannot be sold 12 . We have almost no understanding of why one polymorph forms and not another. We cannot directly observe the process of crystal nucleation, and we have very little quantitative data on the nucleation of competing polymorphs. Here, we provide quantitative nucleation data, use that data to predict how to improve polymorph purity, and successfully test this prediction. We also study crystal growth, and find that its rate depends on nucleation time; crystals of a given polymorph that nucleate at late times are different from those that nucleate at early times. This observation of a link between crystal properties and nucleation time is novel, and potentially allows the control of crystal properties via nucleation.

Our work is a contribution to a growing literature that studies nucleation quantitatively 12 . In a previous publication, 10 , we measured glycine's nucleation rate but did not quantitatively study the competition between glycine's polymorphs. All earlier quantitative work on crystal nucleation, with the exception of that of Diao et al $!^{[5}$, did not consider polymorphism. Diao et al ${ }^{[5}$ studied the polymorphs of a molecule called ROY and found that

\footnotetext{
a) Electronic mail: r.sear@surrey.ac.uk
}

one polymorph dominated nucleation at early times, but competing polymorphs were more likely to nucleate at later times.

We report the results of large-scale (hundreds of nucleation events), quantitative studies of the nucleation of crystals of glycine. As nucleation is a random process, studying just one sample is not enough to characterise a nucleation rate ${ }^{4}$. So we and others study hundreds of samples ${ }^{4|9| 10}$, at each set of conditions. The novelty in our approach lies in our combination of Raman spectroscopy to identify the polymorph of each crystal in situ, with models taken from medical statistics. We show that the statistics of nucleation, and the statistics of mortality are analogous. Both nucleation and death are irreversible processes, and both can have multiple causes, polymorphs in the case of crystal nucleation, competing mortality causes such as lung cancer, heart disease, etc, in the case of death.

\section{A. Previous work on glycine}

We choose glycine because it is a well studied model system for studies of nucleation of crystals from solution $2|3| 6|10| 12|13| 16 \mid 28$. Glycine has three polymorphs: $\alpha, \gamma$ and $\beta$. $\alpha$ glycine is the most common polymorph to crystallise from aqueous solution at neutral $\mathrm{pH}$, but the $\gamma$ polymorph is the most stable of the glycine polymorphs 29 . The $\beta$ polymorph is the least stable. Adding salt favours the $\gamma$ polymorph $3|13| 16|17| 30$.

Glycine also has the advantage that the $\alpha$ and $\gamma$ polymorphs have clearly different Raman spectra ${ }^{5|9| 33}$, in particular in the spectral region between 100 and $200 \mathrm{~cm}^{-1}$, as we can see in Fig. 11(a). The Supplementary Mate- 


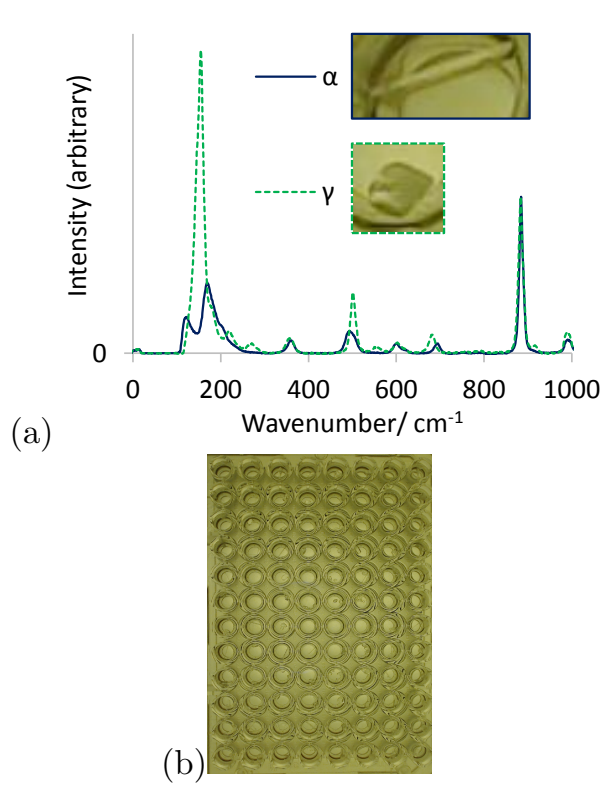

FIG. 1. (a) Raman spectra for $\alpha$ and $\gamma$ crystals, showing that the spectra are distinct. We show a needle-like $\alpha$ crystal and non-needle-like $\gamma$ crystal in the insets. (b) Image of one our 96-well plates, at end of the crystallisation. This is for the first of three runs at a salt concentration of $250 \mathrm{mg} / \mathrm{ml}$. Crystallisation has occurred in 94 wells, with $43 \gamma$ and $51 \alpha$. Each well is circular and has an internal diameter of $6.8 \mathrm{~mm}$.

rial has further details and sets of Raman spectra for both polymorphs. The characteristic peaks of the $\beta$ polymorph are not found. This allows us to use Raman spectroscopy to identify polymorphs. We can do this in situ on crystals a millimetre or less in size, which allows us to identify the polymorphs of the many hundreds of crystals we needed to study to obtain robust statistics. See Fig. 1.(b) for an image of one of the 96-well plates we used to study nucleation in a large number of samples in parallel. We are not the first to use Raman spectroscopy to identify crystals. For example Sultana and Jensen ${ }^{5}$ used it in microfluidic studies of seeded crystallistion, and Cui et al. ${ }^{34}$ used it to study the results of contact-induced nucleation. Diao and coworker $\$ \frac{5}{5}$ have previously identified the polymorph of large numbers of single crystals in situ, but their work relied on the different polymorphs of the molecule ROY having very different visual appearances.

There have been many studies of the growth rate of glycine crystals. Measured growth rates ${ }^{116(35)}$ for glycine vary from $\mu \mathrm{m} / \mathrm{h}$ to $\mathrm{mm} / \mathrm{s}$, a dynamic range of $10^{6}$; see Fig.S1 of the Supplementary Material. Growth rates increase rapidly with increasing supersaturation, and measured growth rates also depend on the experimental set up. The $\alpha$ polymorph consistently grows faster than the $\gamma$ polymorph.

In the next section, we will introduce the statistical tools needed to analyse the nucleation of competing polymorphs. We then have two results sections, one on nucleation and one on crystal growth.

\section{MATERIALS AND METHODS}

Solutions of glycine and sodium chloride were made by adding deionized water (Milli-Q, $18.2 \mathrm{M} \Omega \mathrm{cm}$ ) to solid glycine ( $\geq 99 \%$ HPLC from Sigma, cat. no. G7126) and sodium chloride ( $\geq 99.999 \%$ from Sigma, cat. no. 38979). This solid glycine consisted of mainly the $\gamma$ polymorph but had a small amount of the $\alpha$ polymorph. We fully dissolved the glycine during preparation of the solution. The solution was heated to $70{ }^{\circ} \mathrm{C}$ in a sealed vial and stirred at $1200 \mathrm{rpm}$ for $1 \mathrm{~h}$ using a hot plate and magnetic stirrer bar. At all $\mathrm{NaCl}$ concentrations, the concentration of the glycine solution was fixed at $320 \mathrm{mg}$ of glycine per $\mathrm{ml}$ of water. Solutions were held in the pipette for a time $t_{H}=15 \mathrm{~s}$ before distributing into the wells.

\section{A. Crystallisation experiments}

Each experiment used a new microplate (Nunclon Delta Surface) with 96 wells (arranged as 8 rows of 12 wells, see Fig. 1(b)). Each well initially contained $100 \mu \mathrm{l}$ of tridecane ( $\geq 99 \%$ from Sigma-Aldrich). Then, $100 \mu \mathrm{l}$ of the heated glycine solution was transferred simultaneously into each well in successive rows of wells using a 12-channel multipipette (Scipette). The glycine solution was deposited on top of the tridecane after which it would immediately sink below the oil because of its higher density. The plate and samples cooled to room temperature within the first hour of the experiment. The experiments were carried out in a temperature controlled room, with a setpoint at $21^{\circ} \mathrm{C}$. There are slight variations in temperature during the experiment, as was discussed in our earlier work $k^{10}$, where there are further details of our experimental setup.

\section{B. Determination of nucleation times}

Images of samples were recorded with a Logitech HD Pro Webcam C920 placed underneath the microplate, as in our earlier work ${ }^{10}$. An example image is shown in Fig. 1(b). Images of the microplate were recorded at a resolution of $1080 \times 1920$ pixels. An image was recorded every $10 \mathrm{~min}$ for the first $3 \mathrm{~h}$, then every $30 \mathrm{~min}$ for the following $6 \mathrm{~h}$, and then at every hour for the remainder of the experiment. The images were then analysed by eye to determine the time at which each sample crystallized.

\section{Crystal sizes and growth rates}

We define the size of a crystal as being the largest distance across a crystal, as illustrated in Fig. S2 of our earlier work 10 . The wells are $6.8 \pm 0.2 \mathrm{~mm}$ in diameter, which is 90 pixels in our images, so one pixel is approximately $0.076 \mathrm{~mm}$ across. The smallest crystal we 
observed was two pixels diagonally across. This corresponds to a center-to-center distance of $\sqrt{2} \times 0.076=$ $0.11 \mathrm{~mm}$. This as an approximation of the smallest size at which a crystal can be detected.

We define needle-like crystals as follows. The aspect ratio of crystals can change over time, and initially needle-like crystals may become less needle-like as time progresses. Here, we define needle-like crystals, as crystals that have an aspect ratio above 5:1 for at least the first five hours after nucleation.

\section{Experiments heated after $18 \mathrm{~h}$}

For one set of experiments, we heated the filled microplate 18 hours into each experiment. The microplates were heated in a Sanyo Mov- $112 \mathrm{~F}$ oven. The temperature within the oven was measured to be $30.4{ }^{\circ} \mathrm{C}$, which remained constant to within $0.1^{\circ} \mathrm{C}$. The samples were kept at this temperature in the oven for $48 \mathrm{~h}$. The mass of the samples was measured before and after experiments to check for evaporation of the water (tridecane is much less volatile). The microplate was found to be 0.1 $\mathrm{g}$ lighter after the samples had been in the oven for $48 \mathrm{~h}$. This corresponds to evaporation of $1 \%$ of the total mass of glycine solution.

\section{E. Raman spectroscopy of glycine}

To identify the polymorph of a crystal, we used an NTEGRA Raman microscope (NTEGRA, NT-MDT) equipped with a 20x objective lens, and a $473 \mathrm{~nm}$ laser. The laser exposure time was $20 \mathrm{~s}$ for all samples. The crystals remained in solution within the wells while the Raman spectroscopy was carried out. If a well contained more than one crystal, we obtained spectra from two crystals in the well. The two spectra were always identified as the same polymorph. Raman spectra, together with the XRD patterns we used to validate our use of Raman spectra to identify polymorphs, are in the Supplementary Material.

\section{STATISTICS OF THE NUCLEATION OF COMPETING POLYMORPHS}

Our droplets of supersaturated solution are sufficiently small that in each droplet we only ever observe the nucleation of one polymorph, either $\alpha$ or $\gamma$. In other words nucleation of the two competing polymorphs are mutually exclusive events. Thus each droplet contributes a time and a polymorph, e.g., nucleation after $2 \mathrm{~h}$, of the $\alpha$ polymorph. Our data is therefore of exactly the form studied over many years by medical statisticians who study competing causes of mortality 14 . Their data is also a time and a label, e.g., death 32 weeks after surgery, due to heart disease, death after 22 weeks, due to cancer, etc.
Medical statisticians have developed powerful techniques and best practices for analysing data of this form. We propose that the study of competing polymorphs will benefit greatly from using the methods developed in medical statistics. The study of competing irreversible processes is called survival data analysis20]42]43. Survival here means the survival either of a patient, or here of the solution state, i.e., survival until death or nucleation. Here we introduce the standard way of modelling data of our form.

Survival data in the presence of competing processes are typically plotted as what are called cumulative incidence functions (CIFs) $17 \mid 20$. We record two CIFs, one for the $\alpha$ polymorph, $I_{\alpha}$, and one for the $\gamma$ polymorph, $I_{\gamma}$. The CIF $I_{i}(t)$ is defined as the probability that nucleation has occurred at or before time $t$, and that polymorph $i$ has nucleated. The survival probability $P(t)$ is the probability that nucleation has not occurred at or before time $t$, so $P(t)=1-I_{\alpha}(t)-I_{\gamma}(t)$.

The effective nucleation rates for the two polymorphs, $h_{\alpha}(t)$ and $h_{\gamma}(t)$, are examples of a type of function called cause-specific hazard functions (CSHs) $17 / 20$. The CSH for the nucleation of polymorph $i, h_{i}$, is 1719

$$
h_{i}(t)=\frac{1}{P(t)} \frac{\mathrm{d} I_{i}(t)}{\mathrm{d} t} \quad i=\alpha, \gamma
$$

In words: the effective nucleation rate of a polymorph is the time derivative of the polymorph's CIF, divided by the fraction of wells remaining uncrystallised, $P(t)$.

Both CIFs and CSHs are observables: CIFs are what we measure, and CSHs are time derivatives of the CIFs. It is important to note that although we refer to $h_{\alpha}$ as an effective nucleation rate for the $\alpha$ polymorph, $h_{\alpha}$ also depends on $\gamma$ nucleation. We only observe one polymorph in a well, so once a $\gamma$ crystal has formed in a well, $\alpha$ nucleation will not occur. The two competing processes are entangled, we cannot separate them out, and so care is needed in interpreting $h_{\alpha}$ and $I_{\alpha}$. This problem is discussed by Geskus ${ }^{20}$ and by Beyersmann et al. 17 .

\section{Models that include only observables}

As CSHs are observables, if we directly model these then we have a model that uses only experimentally observable functions. We model the CSHs (the effective nucleation rates) as power laws, with exponents $\beta_{i}$ and characteristic timescales $\tau_{i}$. These CSHs have the form of the hazard function of the widely used ${ }^{\sqrt{4}}$ Weibull function:

$$
h_{i}(t)=\beta_{i}\left(t^{\beta_{i}-1} / \tau_{i}^{\beta_{i}}\right) \quad i=\alpha, \gamma
$$

This model includes the time-independent nucleation rate as a special case $\left(\beta_{i}=1\right)$. 


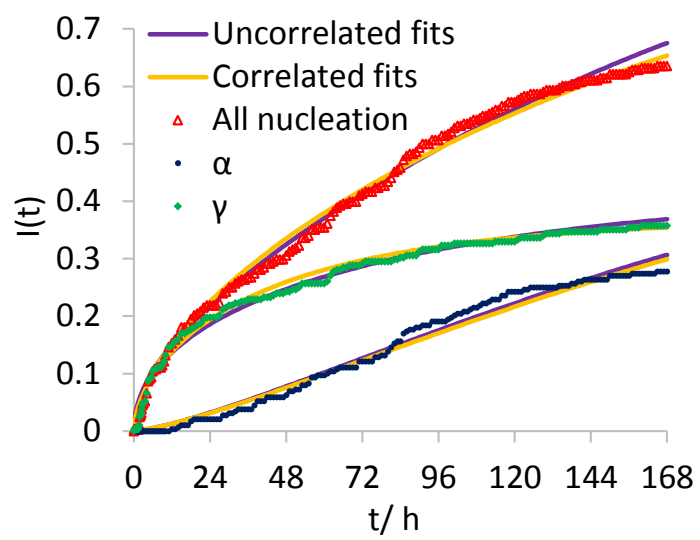

FIG. 2. Plot of CIFs at the salt concentrations of $250 \mathrm{mg} / \mathrm{ml}$. The points are our data: $I_{\alpha}$ (blue), $I_{\gamma}$ (green) and $I_{\alpha}+I_{\gamma}$ (red), respectively. Purple curves are fits to a model in which the values of $t_{n u c, \alpha}$ and $t_{n u c, \gamma}$ for a single well are uncorrelated, and are taken from a Weibull distribution. This is equivalent to a fit to Weibull CSHs. The yellow curves are from a fit of a model in which the values of $t_{n u c, \alpha}$ and $t_{n u c, \gamma}$ for a single well are correlated with a Spearman's rank coefficient of 0.95 .

\section{A. Models with latent nucleation times}

Another approach to modelling our competing nucleation processes is to associate two nucleation times with every droplet $\frac{14-17 \mid 20}{2}$ : a nucleation time for the $\alpha$ polymorph, $t_{n u c, \alpha}$, and a nucleation time for the $\gamma$ polymorph, $t_{n u c, \gamma}$. We only observe the shorter of these two times. The first nucleation event preempts nucleation of the other polymorph, and so we do not observe the longer time. This longer time is then latent, i.e., hidden, and so is not an experimental observable.

Models with latent nucleation times are natural choices for studying possible correlations between the competing processes, and hence for making predictions of what we would observe if we could somehow prevent one polymorph nucleating. Correlations would arise if both polymorphs are nucleating on the same impurity, or if the less stable polymorph forms as a transient intermediate to the formation of a more stable polymorph.

\section{RESULTS FOR THE COMPETING NUCLEATION OF GLYCINE'S POLYMORPHS}

\section{A. Time dependence of the nucleation rates}

In Fig. 2, we have plotted the CIFs for experiments at a salt concentration of $250 \mathrm{mg} / \mathrm{ml}$. The $\alpha$ and $\gamma$ nucleation rates have opposite time dependencies: $\gamma$ nucleation starts fast, $I_{\gamma}$ has an initial steep rise, but then it slows, while $\alpha$ nucleation starts slow but accelerates. Toward the end of the experiment, $I_{\alpha}$ almost catches up with $I_{\gamma}$.
To quantify the variation in the nucleation rates with time, we have fitted models with Weibull-type CSHs (equation (2) ) to the experimental CIF curves. The bestfit parameter values are plotted in Fig. 3, the numbers are in Table S2. A value of $\beta<1$ gives an effective nucleation rate that slows down as time passes, while $\beta>1$ means that the rate accelerates with time. At $250 \mathrm{mg} / \mathrm{ml}$ salt, $\beta_{\gamma}=0.48$ and $\beta_{\alpha}=1.41$, which confirms that $\gamma$ nucleation slows with time while $\alpha$ nucleation accelerates.
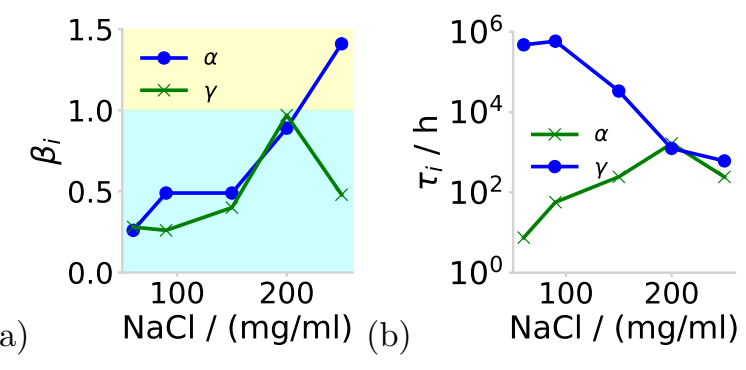

FIG. 3. The Weibull exponents $\beta_{i}$ (a), and $\tau_{i}$ (b) for fits to the nucleation rates of both polymorphs, as functions of the concentration of $\mathrm{NaCl}$. In (a) we have shaded in yellow the region where the nucleation rate increases with time, and in blue the region where it decreases.

The nucleation rate for the $\alpha$ polymorph only accelerates with time at high salt. At low salt the effective nucleation rate for $\alpha$, the $\mathrm{CSH} h_{\alpha}$, actually decreases with time; the best-fit values of $\beta_{\alpha}$ in Fig. 3 are less than one except at the highest salt concentration. Increasing the salt concentration not only slows down the timescale for $\alpha$ nucleation, $\tau_{\alpha}$, it also qualitatively changes the kinetics of nucleation, from initially fast nucleation that slows, to initially slow nucleation that accelerates. Plots of CIFs for five salt concentations are shown in Fig. S7 of the Supplementary Material. Increasing the salt concentration dramatically decreases the apparent timescale for $\gamma$ nucleation, as shown in Fig. 3(b). At high salt concentrations a larger fraction of the crystals are of $\gamma$ polymorph (Fig. S8).

We note that our lowest salt concentrations of 60 $\mathrm{mg} / \mathrm{ml}$ significantly accelerates nucleation with respect to no added salt, which is why we work at a glycine concentration of $320 \mathrm{mg} / \mathrm{ml}$, lower than the $333 \mathrm{mg} / \mathrm{ml}$ where we obtained most of our data in our earlier work 10 . But then at higher salt concentrations the rate slows down again. The nucleation rate does not vary monotonically with salt concentration.

The CIFs in Fig. 2 are obtained by adding together three individual runs. The CIFs for individual runs are shown in Fig. S9(e) in the Supplementary Information. The shapes of the CIFs for individual runs are the same as in Fig. 2, so our conclusions on the time dependencies of $\alpha$ and $\gamma$ nucleation are highly reproducible. There is however significant run-to-run variability in the total amount of nucleation we observe, and to a lesser extent in the fractions of the polymorphs that form. This was also found in earlier work ${ }^{10}$ and is common in crystal nucle- 
ation. For the three runs that make up the data in Fig. 2 the final fractions of the crystals that formed that were of the $\gamma$ polymorph that form are $47 \%, 40 \%$ and $72 \%$, while the final fraction of wells where crystallisation occurred was $98 \%, 45 \%$ and $48 \%$. Reproducibility is comparable at other salt concentrations, see Supplementary Material.

\section{B. We cannot determine if the nucleation of the $\alpha$ and $\gamma$ polymorphs is correlated}

One obvious question about the nucleation of the competing polymorphs is: Are $\alpha$ and $\gamma$ nucleation correlated? By correlated, we mean that if in an individual well the $\alpha$ polymorph is likely to nucleate early, is the $\gamma$ polymorph also likely to nucleate early? We expect that the nucleation in our samples is heterogeneous, i.e., occurring on impurities, and so if the same impurities tend to induce nucleation of both polymorphs, we would expect nucleation of the polymorphs to be correlated.

TABLE I. The best-fit values for fits of Fig. 2 Note that the uncorrelated values refer to both the case of uncorrelated latent nucleation times, and to the case of Weibull CSHs, the two cases are mathematically equivalent. $R_{i}^{2}, i=\alpha, \gamma$, is the $R^{2}$ value for comparison of the fit $I_{i}$ to the data.

\begin{tabular}{c|cccc|cc} 
& $\beta_{\alpha}$ & $\beta_{\gamma}$ & $\tau_{\alpha}(\mathrm{h})$ & $\tau_{\gamma}(\mathrm{h})$ & $R_{\alpha}^{2}$ & $R_{\gamma}^{2}$ \\
Uncorrelated & 1.41 & 0.48 & 244 & 610 & 0.99 & 0.99 \\
\hline Correlated & 0.92 & 0.60 & 164 & 260 & 0.98 & 0.99
\end{tabular}

Unfortunately, Tsiatis $\frac{14}{14}$ has rigorously proved that from data of our form (nucleation time plus polymorph for each well), we cannot determine if the competing processes are independent of each other, or are correlated. We have illustrated this in Fig. 2, where there are fits of our data using models with and without correlations. The two fits are essentially as good as each other; the $R^{2}$ values in Table $\mathrm{I}$, are almost identical. The model with correlations was based on latent nucleation times; details are in the Supplementary Information.

We can see in Table I that when the hazard functions are correlated, the best fit $\beta$ and $\tau$ parameters are significantly changed. Both $\beta_{\alpha}$ and $\beta_{\gamma}$ are now less than one, so if there are strong correlations, the data is consistent with both nucleation rates decreasing with time. We do not know the degree of correlation between $\alpha$ nucleation and $\gamma$ nucleation, and multiple $\beta$ and $\tau$ parameters will fit the data equally well, but with different correlations. This means that with data of this type, we can say almost nothing about what we would observe in the hypothetical cases where we could prevent nucleation of one polymorph, and just observe nucleation of the other ${ }^{14}[1720$.

\section{Polymorph composition vs time}

At high salt, the two hazard functions $h_{\alpha}$ and $h_{\gamma}$ have opposite time dependencies, which implies that the poly-

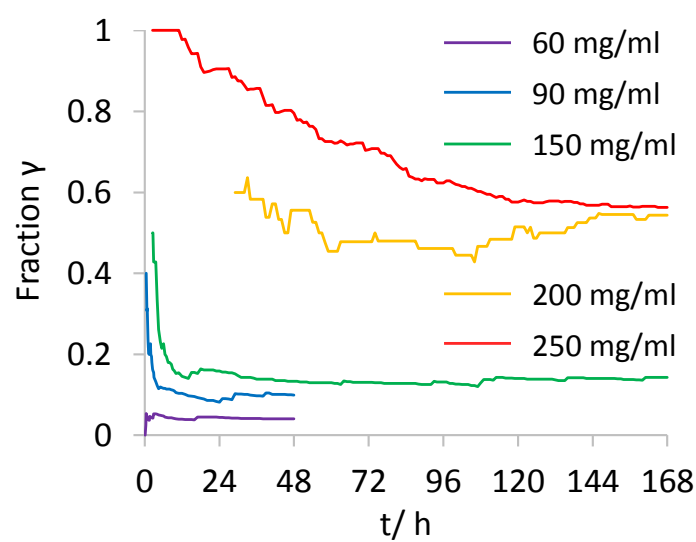

FIG. 4. Polymorph composition as a function of time. The composition is the fraction of the wells where crystallisation has occurred, that contain the $\gamma$ polymorph. The lines start at the point when a total of ten nucleation events have occurred. Each curve is obtained from all runs at that salt concentration.

morph composition varies with time. We have plotted the polymorph composition as a function of time in Fig. 4. We plot $n_{\gamma}(t) /\left[n_{\alpha}(t)+n_{\gamma}(t)\right]$, where $n_{\alpha}(t)$ and $n_{\gamma}(t)$ are the number of wells containing $\alpha$ crystals and $\gamma$ crystals at time $t$, respectively.

If we consider the curve for $250 \mathrm{mg} / \mathrm{ml} \mathrm{NaCl}$, we see that the fraction of $\gamma$ starts at one (pure $\gamma$ ) and decreases towards its final value of 0.56 . This is consistent with our Weibull fits that have exponents $\beta_{\alpha}>\beta_{\gamma}$ (see Fig. 3), and so give a $h_{\alpha}(t) / h_{\gamma}(t)$ that is an increasing function of time. We have many fewer $\gamma$ nucleation events at other salt concentrations, so our conclusions are less reliable there, but it seems likely that at all salt concentrations the fraction of the $\gamma$ polymorph decreases with time. We are not the first to observe a polymorph purity that changes with the time, Diao et al $\stackrel{5}{5}$ found this behaviour for the molecule ROY (see their Figure 8).

\section{Time-dependent supersaturation increases polymorph purity}

When producing crystals, the aim is often to obtain a particular polymorph with high purity. We observed that at a salt concentration of $250 \mathrm{mg} / \mathrm{ml}, \gamma$ glycine nucleation dominated at early times, but then the $\alpha$ nucleation rate increased. This suggests that stopping nucleation early in the experiment will increase $\gamma$-glycine purity. To test this hypothesis we performed additional experiments at $250 \mathrm{mg} / \mathrm{ml} \mathrm{NaCl}$. In these experiments we reduced the supersaturation after 18 hours, by increasing the temperature from room temperature $\left(21^{\circ} \mathrm{C}\right)$ to $30.4{ }^{\circ} \mathrm{C}$. This had the effect of almost completely halting nucleation after 18 hours; in two runs only one crystal nucleated after 18 hours.

The final fraction of crystals that are in the $\gamma$ poly- 


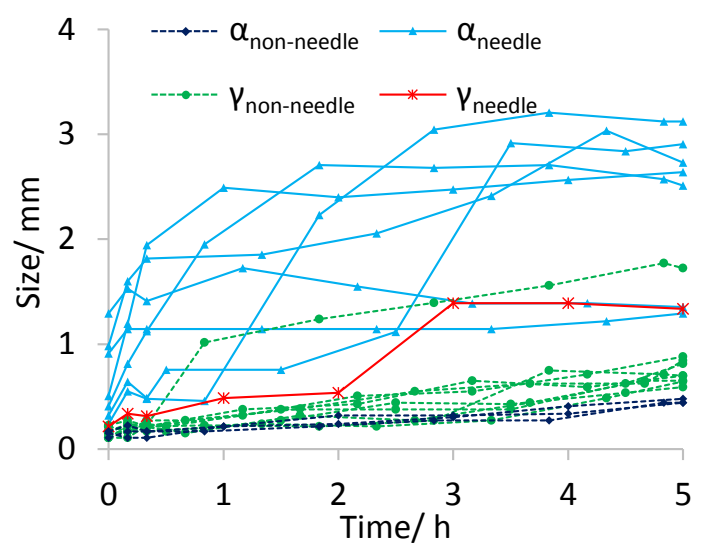

FIG. 5. Plot of the maxium distance across a crystal, as a function of time since it nucleated. Data is shown for ten $\alpha$ and ten $\gamma$ crystals. These are seven $\alpha$ needle crystals plus three $\alpha$ non-needle crystals, and one $\gamma$ needle plus nine $\gamma$ non-needles. For each crystal $t=0$ is defined as the time of the first image in which there is a visible crystal. The salt concentration is $250 \mathrm{mg} / \mathrm{ml}$.

morph increases from $0.56 \pm 0.04$ to $0.94 \pm 0.02$. Here, the first number is the final fraction of $\gamma$ crystals at the end $(168 \mathrm{~h})$ of three isothermal runs, and the second number is the fraction of $\gamma$ crystals after $18 \mathrm{~h}$, of all five runs. The CIFs for these experiments are in Fig. S10.

\section{RESULTS FOR THE GROWTH RATES AND CRYSTAL HABITS OF GLYCINE CRYSTALS}

Having considered nucleation, we will now turn to consider crystal growth. We will present results that show that nucleation and growth are linked. We have plotted the sizes of $10 \alpha$ and $10 \gamma$ crystals, as a function of time, in Fig. 5 .

Note that we estimate the size of a crystal, by using the maximum length across the crystal; see the Materials and Methods section, and our earlier work $\frac{10}{10}$ for details of how we measure size. Some crystals grow as needlelike shapes, while others have more compact habits. We show example crystals with needle and non-needle habits in Fig. 6.

Three things are clear from Fig. 5. i) The growth rates of the linear dimension of both polymorphs vary between one crystal and another. ii) Much of this variability is related to crystal habit. Needle-like crystals have much faster growth rates along their long axis than do nonneedle-like crystals. iii) Growth of the $\alpha$ polymorph is generally faster than the $\gamma$ polymorph (in agreement with other studies ${ }^{2}$ ). Note that growth of the needles perpendicular to their long axis is quite slow, approximately comparable to the growth rate of non-needle-like crystals. Therefore, although one axis of the needles grows very rapidly, the growth of the volume of these crystals may not be faster than the growth of the volume of the non-needle-like crystals.

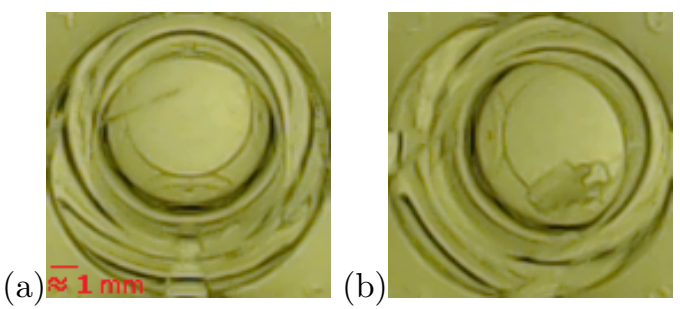

FIG. 6. Here we show some typical crystal habits. (a) is a needle-like $\alpha$ crystal, observed less than an hour after nucleation. (b) is a non needle-like $\alpha$ crystal observed $5 \mathrm{~h}$ after nucleation. The scale bar in (a) applies to both images.

We do not know why the growth rate of crystals growing under identical conditions is so variable. Crystal growth often relies on defects $\frac{46}{\text {, }}$, and so it may be due to different crystals acquiring different defects as they grow. But whatever the cause, this variability has consequences. For example, any crystallisation model that assumes a single well-defined growth rate at a given supersaturation is clearly very far from the truth for glycine.

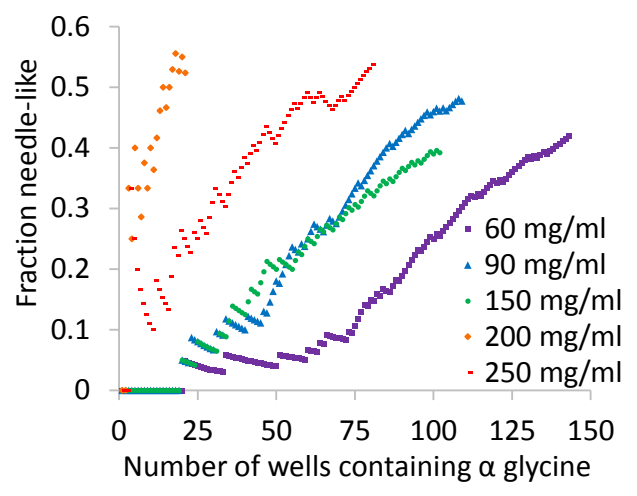

FIG. 7. For the $\alpha$ polymorph, we plot the fraction of crystals with a needle-like habit, as a function of the total number of wells that contain $\alpha$ crystals.

\section{A. Nucleation time and crystal habit}

Next we look at whether the habit of a crystal depends on the time at which it nucleates. In Fig. 7 we plot the fraction of crystals of the $\alpha$ polymorph that are needlelike, as a function of the total number of wells with $\alpha$ crystals. The $x$-axis is effectively a time axis, as the number crystallised increases with time, but using the number crystallised as the $x$-axis allows easier comparison between different salt concentrations.

At early times almost all crystals have the non-needlelike habit. However, at later times most crystals that nucleate are needles, so the fraction of needle-like crystals increases rapidly. There is a very strong dependence of crystal habit on nucleation time. This is due to these 
late-nucleating crystals having much faster growth along one axis (only). For example at $60 \mathrm{mg} / \mathrm{ml}$, for the first $70 \alpha$ nucleation events, six are needle-like crystals, but for the following 70 nucleation events, 51 are needle-like. For $\gamma$ crystals at high salt concentrations, we see the same trend, see Fig. S14, but as we have few needle-like $\gamma$ crystals, our statistics are poorer.

We were surprised when we found that typical latenucleating crystals have a very different growth rate along one axis than typical early-nucleating crystals. We analysed the Raman spectra for needles and non-needles, see Supplementary Material. Although there is crystalto-crystal variability in the Raman spectra, there is no sign of any systematic difference between the set of the spectra of needles, and the set of spectra of the nonneedles. So via Raman spectroscopy we separated our crystals into two sets: one set with spectra chracteristic of the $\alpha$ polymorph, and one with spectra characteristic of the $\gamma$ polymorph 9 . Both sets of crystals contain needles and non-needle-like crystals. Our XRD patterns (Fig. S3(a) and Fig. S4) confirm this finding.

Finally, note that in these XRD patterns we only ever observe peaks characteristic of the $\alpha$ and $\gamma$ polymorphs; characteristic peaks for $\beta$ are absent (Fig. S4). In addition, the $\beta$ polymorph is not typical for crystallisation from aqueous solutions, and indeed under these conditions is expected to be unstable with respect to conversion to the $\alpha$ form 47 . We do not observe visible changes in habit that might suggest polymorph-to-polymorph conversion.

We do not know what causes the growth and habit difference between early- and late-nucleating crystals. However, if it is due to differences in defects that control crystal growth, then this suggests that nucleation may involve more than one step, and that at least one of these rate limiting steps involves nucleation of a growth defect. It is possible that our initially slow nucleation of the $\alpha$ polymorph at high salt concentration is associated with slow or multistep defect nucleation, which is needed before that polymorph can grow.

\section{CONCLUSIONS}

We have studied the competing nucleation of two crystal polymorphs of glycine, using Raman spectroscopy to identify the polymorph of individual crystals. We found that at high salt, the nucleation behaviour of the competing polymorphs is analogous to the competition of the tortoise and the hare in Aesop's story. In the story, at first the hare runs faster than the tortoise, but then the hare goes to sleep, allowing the tortoise to overtake it. Like the hare, $\gamma$ nucleation is initially fast but then slows, while $\alpha$ nucleation accelerates during the experiment, and so the rate of $\alpha$ nucleation overtakes that of $\gamma$ nucleation.

This observation can be exploited. Drawing on this observation, we predicted that reducing the supersaturation after $18 \mathrm{~h}$ would increase the purity of the $\gamma$ polymorph. It did, the fraction of the $\alpha$ impurity was reduced from $44 \%$ to $4 \%$. Thus, the complex time dependence of competing polymorphs can be exploited to greatly increase the purity of a polymorph.

By using a result from medical statistics 14 , we were able to prove that with our data it is impossible to disentangle the nucleation of $\alpha$ and $\gamma$ polymorphs $14 \mid 17 / 20$. These two processes may or may not be strongly correlated - we simply do not know. This means that care must be taken when interpreting results on competing polymorphs. For example, if we alter an experimental parameter, such as salt concentration, $\mathrm{pH}$, etc, and observe more of one polymorph, that does not allow us to infer that the nucleation rate of that polymorph has increased, the rate of the competing polymorph may have decreased 16 . Work by medical statisticians over the last 40 years has found that interpretation of data of our form is subtle, and so we will need to learn from their work, in order to correctly understand the competition between polymorphs.

A possible way around this problem is to use the experimental approach of Laval et al!11, who cycled their system, to repeatedly induce crystallisation and dissolution of crystals, in a set of droplets produced by microfluidics. They did not consider polymorphism, but if their approach can be applied to a system with competing polymorphs, then the two competing processes could then be disentangled.

All our results are obtained from system volumes of $0.1 \mathrm{ml}$, and in common with most earlier work, we do not know how varying the volume will change what is seen. However, we do note that our droplets are large enough that we often observe two or more crystals, and that in all cases studied (via Raman) all the crystals in the same droplet are of the same polymorph. This strongly suggests that the nucleation events that produce multiple crystals in a single well are not independent. The second, third, etc, crystals may be forming via secondary nucleation $48[49$, i.e., arising due to the already-existing crystal.

In addition to nucleation, we also studied crystal growth. For both polymorphs we observed fast growing needle-like crystals, and much slower growing nonneedle-like crystals. Nucleation time, crystal habit and growth rate are all correlated. Crystals that nucleate at late times tend to have needle-like habits, and to grow rapidly. This appearance of the fastest growing crystals at late times is the opposite to what we would expect. Further work will be needed to understand the mechanism that underlies this interesting observation.

\section{SUPPLEMENTARY MATERIAL}

The Supplementary Material contains: (I) Literature data on the growth rates of glycine crystals. (II) The solubility of glycine in water, as a function of temperature. 
(III) Raman spectra and XRD patterns of our crystals, as well as reference XRD patterns of all three polymorphs, for comparison. (IV) Details of the statistical techniques we borrow from the field of medical statistics, to model our data. (V) Additional experimental results for nucleation. (VI) Additional experimental results for growth.

\section{ACKNOWLEDGMENTS}

We thank Violeta Doukova (Surrey) for technical assistance, Dan Driscoll (University of Surrey) for help with the X-ray diffraction, and Sharon Cooper (University of Durham), Baron Peters (University of California Santa Barbara) and Jan Sefcik (University of Strathclyde) for helpful discussions. We acknowledge EPSRC for funding a PhD studentship for LJL.

\section{DATA AVAILABILITY}

The authors confirm that data underlying the findings are available without restriction at https://doi.org/10.6084/m9.figshare.c.3898480.

${ }^{1}$ J. Bauer, S. Spanton, R. Henry, J. Quick, W. Dziki, W. Porter, and J. Morris, Pharma. Res. 18, 859 (2001)

${ }^{2}$ S. L. Morissette, S. Soukasene, D. Levinson, M. J. Cima, and O. Almarsson, Proc. Nat. Acad. Sci. 100, 2180 (2003).

${ }^{10}$ L. J. Little, R. P. Sear, and J. L. Keddie, Crys. Growth Design 15, 5345 (2015).

${ }^{4}$ R. P. Sear, CrystEngComm 16, 6506 (2014).

${ }^{5}$ Y. Diao, K. E. Whaley, M. E. Helgeson, M. A. Woldeyes, P. S. Doyle, A. S. Myerson, T. A. Hatton, and B. L. Trout, J. Am. Chem. Soc. 134, 673 (2012)

${ }^{\circ}$ A. I. Toldy, A. Z. M. Badruddoza, L. Zheng, T. A. Hatton, R. Gunawan, R. Rajagopalan, and S. A. Khan, Crys. Growth Des. 12, 3977 (2012).

${ }^{7}$ J.-W. Kim, J.-H. Park, H.-M. Shim, and K.-K. Koo, Crys. Growth Des. 13, 4688 (2013)

${ }^{8}$ C. Brandel and J. H. ter Horst, Faraday Discuss. 179, 199 (2015)

${ }^{9}$ S. Akella, A. Mowitz, M. Heymann, and S. Fraden, Crys. Growth Design 14, 4487 (2014).

${ }^{10}$ D. Duft and T. Leisner, Atmos. Chem. Phys. 4, 1997 (2004).

${ }^{11}$ P. Laval, A. Crombez, and J.-B. Salmon, Langmuir 25, 1836 (2009).

${ }^{12}$ N. Javid, T. Kendall, I. S. Burns, and J. Sefcik, Crystal Growth \& Design 16, 4196 (2016).

${ }^{13}$ X. Yang, J. Lu, X.-J. Wang, and C.-B. Ching, J. Cryst. Growth 310, 604 (2008)

${ }^{2}$ G. Han, P. S. Chow, and R. B. H. Tan, Crys. Growth Design 12, 2213 (2012).

${ }^{3}$ G. Han, P. S. Chow, and R. B. H. Tan, Cryst. Growth Design 15, 1082 (2015).

${ }^{16} \mathrm{~K}$. Srinivasan and J. Arumugam, Optical Materials 30, 40 (2007)

${ }^{17}$ K. Srinivasan, J. Cryst. Growth 311, 156 (2008)

${ }^{18}$ K. Kim, A. Centrone, T. A. Hatton, and A. S. Myerson, CrystEngComm 13, 1127 (2011)

${ }^{19}$ C. Forsyth, I. S. Burns, P. A. Mulheran, and J. Sefcik, Cryst. Growth Design 16, 136 (2016).

${ }^{20}$ S. K. Poornachary, P. S. Chow, and R. B. H. Tan, Crys. Growth Des. 8, 179 (2008).

${ }^{21}$ J. W. Chew, S. N. Black, P. S. Chow, R. B. H. Tan, and K. J. Carpenter, CrystEngComm 9, 128 (2007).
${ }^{22}$ C. Chen, O. Cook, C. E. Nicholson, and S. J. Cooper, Cryst. Growth Design 11, 2228 (2011).

${ }^{23}$ A. Jawor-Baczynska, B. D. Moore, H. S. Lee, A. V. McCormick, and J. Sefcik, Faraday Discuss. 167, 425 (2013)

${ }^{24}$ G. Han, S. Thirunahari, P. Shan Chow, and R. B. H. Tan, CrystEngComm 15, 1218 (2013).

${ }^{25}$ G. He, V. Bhamidi, S. R. Wilson, R. B. H. Tan, P. J. A. Kenis, and C. F. Zukoski, Crys. Growth Design 6, 1746 (2006).

${ }^{26}$ K. Kim, I. s. Lee, A. Centrone, T. A. Hatton, and A. S. Myerson, J. Am. Chem. Soc. 131, 18212 (2009)

${ }^{27}$ C. E. Nicholson, S. J. Cooper, C. Marcellin, and M. J. Jamieson, J. Am. Chem. Soc. 127, 11894 (2005).

${ }^{28}$ C. E. Nicholson, C. Chen, B. Mendis, and S. J. Cooper, Crys. Growth Design 11, 363 (2011).

${ }^{29}$ S. A. Rivera, D. G. Allis, and B. S. Hudson, Cryst. Growth Design 8, 3905 (2008).

${ }^{30}$ N. Duff, Y. R. Dahal, J. D. Schmit, and B. Peters, J. Chem. Phys. 140, 014501 (2014).

${ }^{9}$ Y. Shi and L. Wang, J. Phys. D App. Phys. 38, 3741 (2005)

${ }^{5}$ M. Sultana and K. F. Jensen, Cryst. Growth Design 12, 6260 (2012).

${ }^{33}$ J. Baran and H. Ratajczak, Spectrochimica Acta A 61, 1611 (2005)

${ }^{34}$ Y. Cui, J. Stojakovic, H. Kijima, and A. S. Myerson, Crys. Growth Design 16, 6131 (2016).

${ }^{35}$ G. Han, S. K. Poornachary, P. S. Chow, and R. B. H. Tan, Cryst. Growth Design 10, 4883 (2010).

${ }^{1} \mathrm{~L}$. Li and N. Rodrguez-Hornedo, J. Cryst. Growth 121, 33 (1992)

${ }^{4}$ R. Dowling, R. J. Davey, R. A. Curtis, G. Han, S. K. Poornachary, P. S. Chow, and R. B. H. Tan, Chem. Commun. 46, $5924(2010)$

${ }^{14}$ A. Tsiatis, Proc. Nat. Acad. Sci. 72, 20 (1975).

${ }^{15}$ A. V. Peterson, Proc. Nat. Acad. Sci. 73, 11 (1976).

${ }^{16} \mathrm{E}$. Slud and D. Byar, Biometrics 44, 265 (1988).

${ }^{17}$ J. Beyersmann, A. Latouche, A. Buchhol, and M. Schumacher, Statist. Med. 28, 956 (2009).

${ }^{42}$ D. R. Cox and D. Oakes, Analysis of Survival Data (Chapman and Hall, 1984).

${ }^{43}$ E. T. Lee, Statistical Methods for Survival Data Analysis, 2nd ed. (Wiley, 1992).

${ }^{20}$ R. B. Geskus, Data Analysis with Competing Risks and Intermediate States (Chapman and Hall / CRC, 2015).

${ }^{19}$ J. J. Dignam, Q. Zhang, and M. N. Kocherginsky, Clin Cancer Res, 2301 (2012).

${ }^{46}$ R. Davey and J. Garside, From Molecules to Crystallizers (Oxford University Press, Oxford, 2000).

${ }^{47}$ E. S. Ferrari, R. J. Davey, W. I. Cross, A. L. Gillon, and C. S. Towler, Cryst. Growth Design 3, 53 (2003).

${ }^{48}$ G. D. Botsaris, in Industrial Crystallization, edited by J. Mullin (Springer US, 1976) pp. 3-22.

${ }^{49}$ S. G. Agrawal and A. H. J. Paterson, Chem. Eng. Comm. 202, 698 (2015). 


\section{Supplementary Material for: Controlling the crystal polymorph by exploiting the time dependence of nucleation rates}

This supplementary material has six sections:

(I) We discuss literature values of glycine crystal growth rates in the main text. In this section we plot the rates as a function of supersaturation and we give details of the experimental conditions in these studies.

(II) This contains plots of the solubility of glycine in water, as a function of temperature.

(III) This section contains Raman spectra of the $\alpha$ and $\gamma$ polymorphs of glycine. We also show the X-ray diffraction (XRD) results that validate Raman spectroscopy's ability to distinguish between the two polymorphs of glycine.

(IV) Background to the statistics needed to understand the nucleation of competing polymorphs, and details of the models we used.

(V) Additional experimental results for nucleation.

(VI) Additional experimental results for growth.

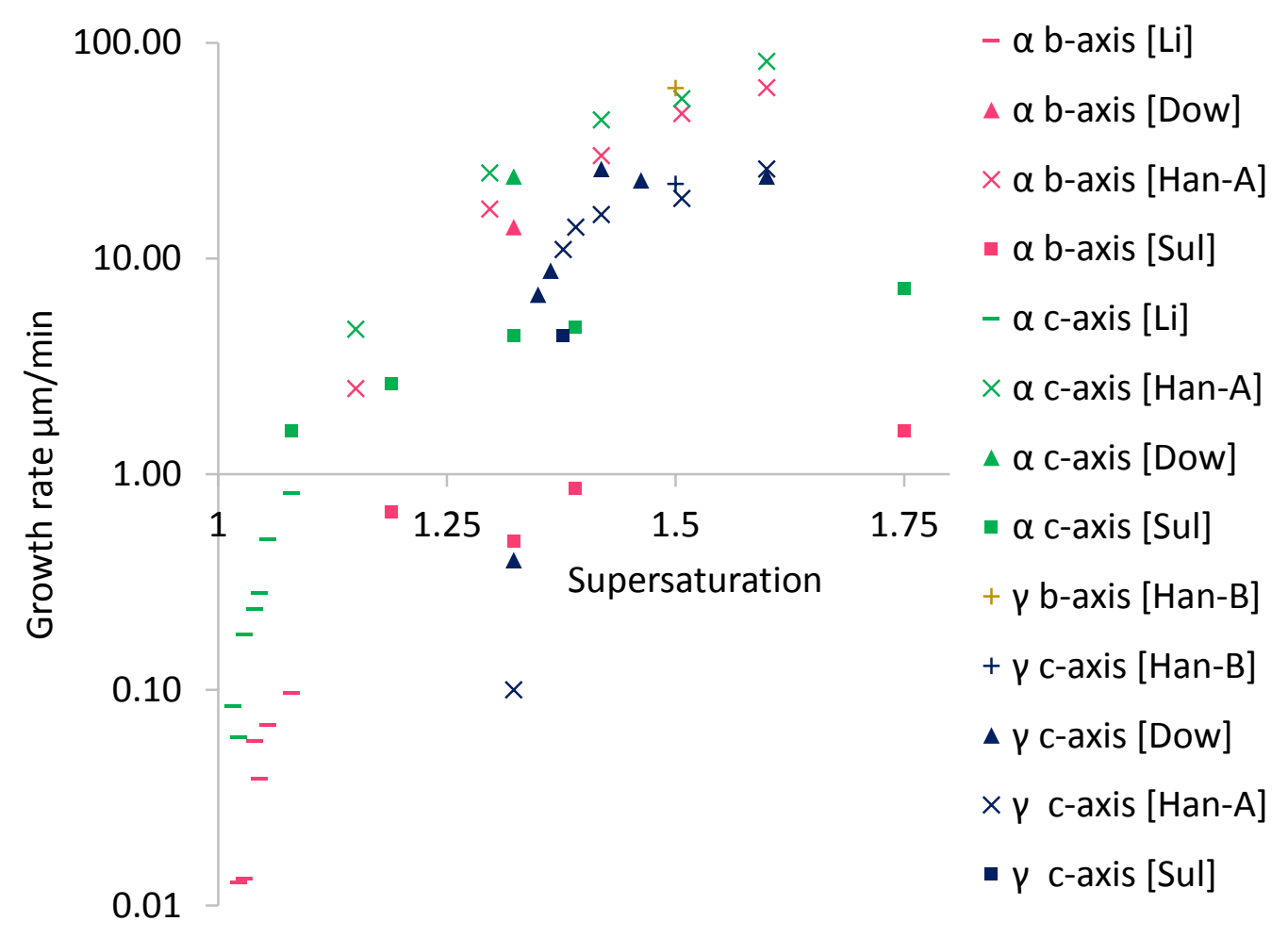

Figure S1. Glycine crystal growth rates, are plotted as a function of supersaturation. This is data from five earlier studies: $[\mathrm{Li}](\mathrm{Li} \text { et al. })^{1},[\mathrm{Han}-\mathrm{A}](\text { Han et al. })^{\sqrt{2}}$, [Han-B] (Han et al. $)^{3}$, [Dow] (Dowling et al. $)^{\sqrt{4}}$ and [Sul] (Sultana et al. $)^{\sqrt{5}}$. These growth rates have been obtained from graphs, so are approximate. Colour coding is as follows, $\alpha$ b-axis, $\alpha$ c-axis, $\gamma$ b-axis and $\gamma$ c-axis are pink, green, gold and dark blue respectively. [Sul] used an anti-solvent, other studies are purely aqueous.

\section{LITERATURE STUDIES OF THE GROWTH OF GLYCINE CRYSTALS}

In the main text we discussed literature values for the growth rates of glycine crystals. These rates are plotted in Figure S1. There was one growth $\mathrm{rate}^{6}$, of $\approx 80,000 \mu \mathrm{m} / \mathrm{min}$, which was too large to fit on our graph. The experimental details for these experiments are summarised in Table S1.

Crystal growth rates appear to be very sensitive to crystal and solution properties, such as crystal size and solvent. The growth rates of $\alpha$ glycine measured by Sultana et al. are lower than those measured by Han et al. and Dowling et 
Table S1. The experimental details of a number of studies on glycine growth. The growth rates from these studies are shown in Figure $\mathrm{S} 1$.

\begin{tabular}{|c|c|c|}
\hline Authors & $\begin{array}{l}\text { Supersaturation } \\
\text { range }\end{array}$ & Experimental set-up \\
\hline Toldy et al 6 & $3.5-6.5$ & $\begin{array}{l}\text { Crystals nucleate in supersaturated droplets within an emulsion at } \\
84^{\circ} \mathrm{C} \text {. Crystals sizes are of the order of tens of } \mu \mathrm{m} \text {. }\end{array}$ \\
\hline Dowling et al. & $1.3-1.45$ & $\begin{array}{l}\text { Individual crystal seeds are placed in supersaturated solution, and ob- } \\
\text { served under a microscope at } 20^{\circ} \mathrm{C} \text {. Crystal sizes are of the order of } \\
\text { mm. Solubility } c_{s} \text { is given as } 212 \mathrm{~g} / \mathrm{l} \text {. }\end{array}$ \\
\hline Han et al! & $1.15-1.6$ & $\begin{array}{l}\text { Individual crystal seeds are placed in supersaturated solution and ob- } \\
\text { served under a microscope at } 23^{\circ} \mathrm{C} \text {. Crystal sizes are of the order of } \\
\text { mm. Solubility } c_{s} \text { is given as } 226 \mathrm{~g} / \mathrm{l} \text {. }\end{array}$ \\
\hline Sultana et al. & $1.08-1.75$ & $\begin{array}{l}\text { Supersaturated solutions containing crystal seeds flow through a PDMS } \\
\text { (poly-dimethylsiloxane) microfluidic device. Solutions are supersatu- } \\
\text { rated using the anti-solvent methanol. Crystal sizes are of the order } \\
\text { tens of } \mu \mathrm{m}\end{array}$ \\
\hline Li et al ${ }^{1}$ & $1.01-1.08$ & $\begin{array}{l}\text { Crystal seeds are placed in a glass cell at } 23^{\circ} \mathrm{C} \text { while supersaturated } \\
\text { solution flows through the cell, seed sizes are of the order of hundreds } \\
\text { of } \mu \mathrm{m}\end{array}$ \\
\hline Han et al. ${ }^{[3}$ & 1.5 & Set-up is the same as in Dowling et al. ${ }^{4}$ \\
\hline
\end{tabular}

al. In the work of Sultana et al., the glycine solution is supersaturated by the addition of the anti-solvent methanol, while in the experiments of Han et al. and Dowling et al. only water and glycine are present. Also, both Dowling and Han work with crystals with sizes of the order of mm, while Sultana et al. work with crystals of sizes of the order tens of $\mu \mathrm{m}$. It may be that the presence of methanol is affecting the growth of crystals in the experiments of Sultana et al, and the crystal growth rate may also be changing with crystal size.

\section{GLYCINE SOLUBILITY}

For glycine in water the solubility varies as shown in Figure S2 We can see that glycine's solubility is very sensitive to temperature. This is beneficial in that it allows us to easily create highly supersaturated solutions (by cooling), but problematic in that to perform an experiment at constant supersaturation, temperature must be controlled very precisely. The curve in Figure $\mathrm{S} 2(\mathrm{a})$, is used for our supersaturation calculations. We include solubility data from several sources in Figure S2 (b) to show that values for glycine solubility in the literature can vary significantly between studies.

\section{VALIDATION OF RAMAN SPECTRA FOR IDENTIFYING THE $\alpha$ AND $\gamma$ POLYMORPHS OF GLYCINE}

The $\alpha$ and $\gamma$ crystals have distinct Raman spectra, which can be identified by a set of distinctive peaks 9 . Examples of spectra from each polymorph are given in Figure S3(b). Both polymorphs have a very intense peak at $886 \mathrm{~cm}^{-1}$. There are clear differences between the two spectra in the region 100-200 $\mathrm{cm}^{-1}$ where the peaks can be attributed to intermolecular vibrations ${ }^{9}$. The $\alpha$ polymorph has several small peaks (relative to the $886 \mathrm{~cm}^{-1}$ peak) at $118 \mathrm{~cm}^{-1}$, $164 \mathrm{~cm}^{-1}, 171 \mathrm{~cm}^{-1}$ and $203 \mathrm{~cm}^{-1}$, which the $\gamma$ polymorph does not have. The $203 \mathrm{~cm}^{-1}$ peak is often of low intensity and the peaks at $164 \mathrm{~cm}^{-1}$ and $171 \mathrm{~cm}^{-1}$ are often merged. Spectra where these peaks are more clearly visible can be seen in Figure S3(c) and (d). The $\gamma$ polymorph has a very intense peak (comparable in intensity to the $886 \mathrm{~cm}^{-1}$ peak) at $157 \mathrm{~cm}^{-1}$, which the $\alpha$ polymorph does not have. There are also differences between the polymorphs at $\approx$ $500 \mathrm{~cm}^{-1}$. The $\alpha$ polymorph has two low intensity peaks at $492 \mathrm{~cm}^{-1}\left(\mathrm{NH}_{3}\right.$ torsional mode $\left.{ }^{9}\right)$ and $502 \mathrm{~cm}^{-1}\left(\mathrm{CO}_{2}\right.$ rocking mode $\mathrm{e}^{9}$ ) while the $\gamma$ polymorph has just one high intensity peak at $504 \mathrm{~cm}^{-1}$.

X-ray diffraction (XRD) was used as a test to confirm the results of our Raman spectroscopy analysis. A sample of crystals was analysed with Raman spectroscopy (the two example spectra shown in Figure S3(b)). Immediately after the Raman spectroscopy had been carried out, the crystals were removed from the microplate and separated into two groups: Those that had been identified as $\alpha$ glycine and those that had been identified as $\gamma$ glycine. The two samples 
(a)

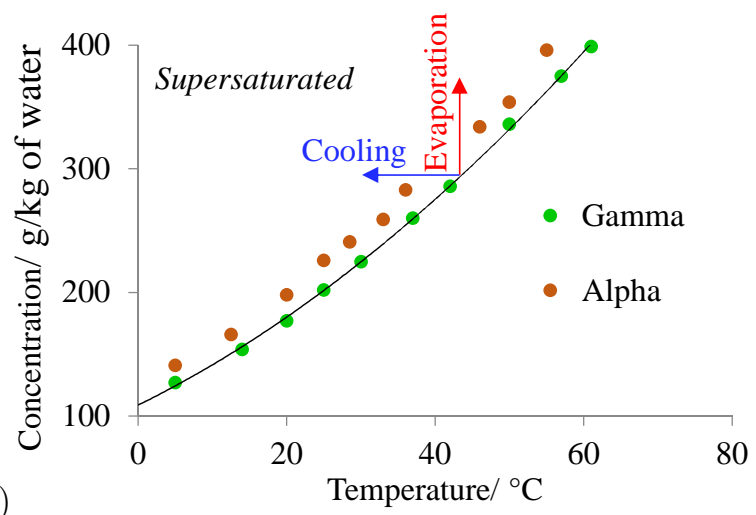

Figure S2. Solubility in water of the $\alpha$ and $\gamma$ polymorphs of glycine as a function of temperature. (a) Data from Yang et a 7 . We have fit a second order polynomial (black curve), so that the solubility can be approximated between data points. This fit is solubility $(\mathrm{g} / \mathrm{kg})=0.0301 T^{2}+2.96 T+109$, with $T$ in ${ }^{\circ} \mathrm{C}$. (b) Comparison of $\gamma$ glycine solubility from several sources: Yang

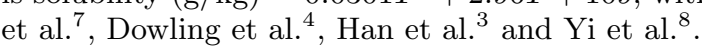

were then prepared for XRD using the same procedure as described in earlier work 10 . The crystals were ground up into a fine powder before analysis. Powder XRD was carried out using a PANalytical XPert Pro diffractometer across a $2 \theta$ range of $10-70^{\circ}$ using $\mathrm{Cu} K_{\alpha}$ radiation. The resulting XRD patterns can be seen in Figure S3. In Figure S3 we have identified peaks as belonging to either the $\alpha$ or $\gamma$ polymorphs using the standard JCPDS XRD patterns of these polymorphs. The $\alpha$ polymorph pattern is JCPDS number 00-032-1702, and the $\gamma$ polymorph is JCPDS number 00-006-0230.

As expected, the set of crystals which we identified as $\alpha$ with Raman spectroscopy are found to show only $\alpha$ glycine peaks with powder XRD. The Raman spectra and XRD were also in agreement for the set of $\gamma$ crystals. We never observed the third polymorph of glycine, $\beta$ glycine, in our XRD patterns. The $\beta$ polymorph can form needle-like crystals 11 , resembling those we do see, but the XRD patterns rule out significant amounts of the $\beta$ polymorph, at the point at the end of the experiment when XRD patterns are taken. In Figure S4 we have plotted our XRD patterns together with reference $\alpha, \gamma$ and $\beta$ patterns. Note that this range of $2 \theta$ includes the strongest peak of the $\beta$ polymorph (at $2 \theta=18.1^{\circ}$ ) which is absent in both our sets of diffractograms.

During the course of our experiments, we do not observe habit changes of our crystals. This suggests that once formed, our crystals do not transform into another polymorph. However, both Raman and XRD analyses are made at the end of our runs, which is days after many of the crystals formed. Thus, although it seems unlikely, we cannot rule out a polymorph-to-polymorph transformation during our experiment that does not affect habit.

\section{A. Raman spectra for needle-like and non-needle-like crystals}

We were surprised by our observation that both polymorphs formed two very different crystal habits, in each case a needle-like habit and a much more compact habit. So we looked carefully at the Raman spectra we obtained from these crystals. Raman spectra for needle-like and non-needle crystals are shown in Figure $\mathrm{S} 5$ for the $\alpha$ polymorph and in Figure S6 for the $\gamma$ polymorph. In Figure S5(a) we see Raman spectra from 50 needle and non-needle-like crystals, of the $\alpha$ polymorph. Figure S5(c) is a scatter plot of estimates of the height of the peak nominally located at $118 \mathrm{~cm}^{-1}$, versus its position, and the height of the combined 164 and $171 \mathrm{~cm}^{-1}$ peaks, versus their location. The peak heights and positions are obtained by fitting the sum of two Gaussians plus a constant background term to the spectra over the range 100 to $200 \mathrm{~cm}^{-1}$. Note that the Raman spectrum of the $\beta$ polymorph is distinct from that of the $\alpha$ and $\gamma$ polymorphs $12\left[13\right.$. The Raman spectrum of the $\beta$ is flat betweeen approximately 120 and $200 \mathrm{~cm}^{-1}$, whereas we see peaks in that range, in both Figures $\mathrm{S} 5$ and $\mathrm{S} 6$.

Note that there is clearly significant variability in the peak height, but that almost all estimates of the peak position are within a narrow range. The distributions of the peak heights and positions of the needle and non-needle crystals are indistinguishable. As far as the Raman spectra are concerned, the needle-like and non-needle-like both have the characteristic $\alpha$ polymorph peaks at the same position, and in both cases with variable intensity. Whatever the cause of the fast growth along one axis of the needles, it is not apparent in the Raman spectra. This would be consistent with the difference in growth rates being due to different defects, as these defects would be unlikely to show up in the Raman spectra. 

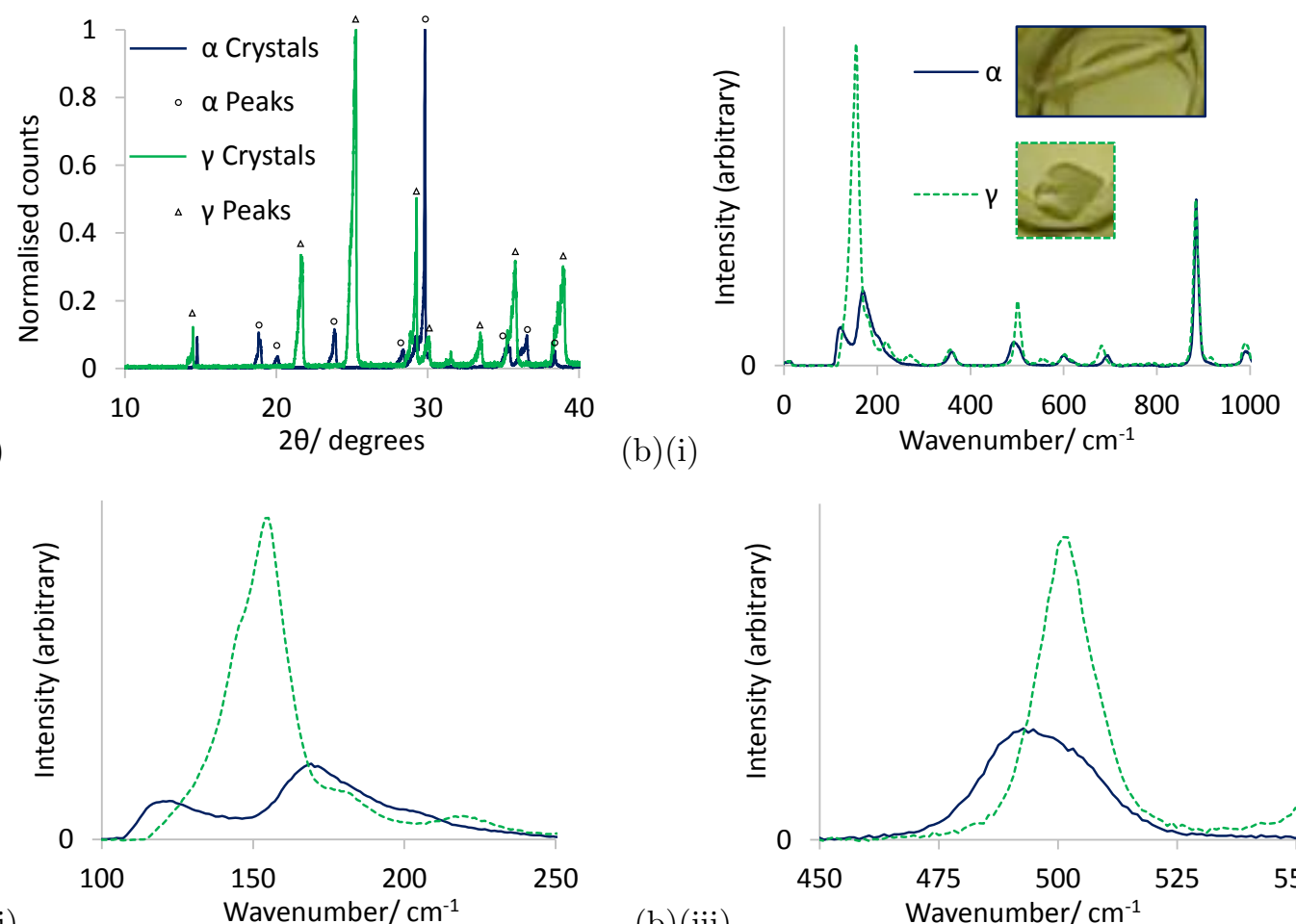

(b)(ii)

(b) (i)
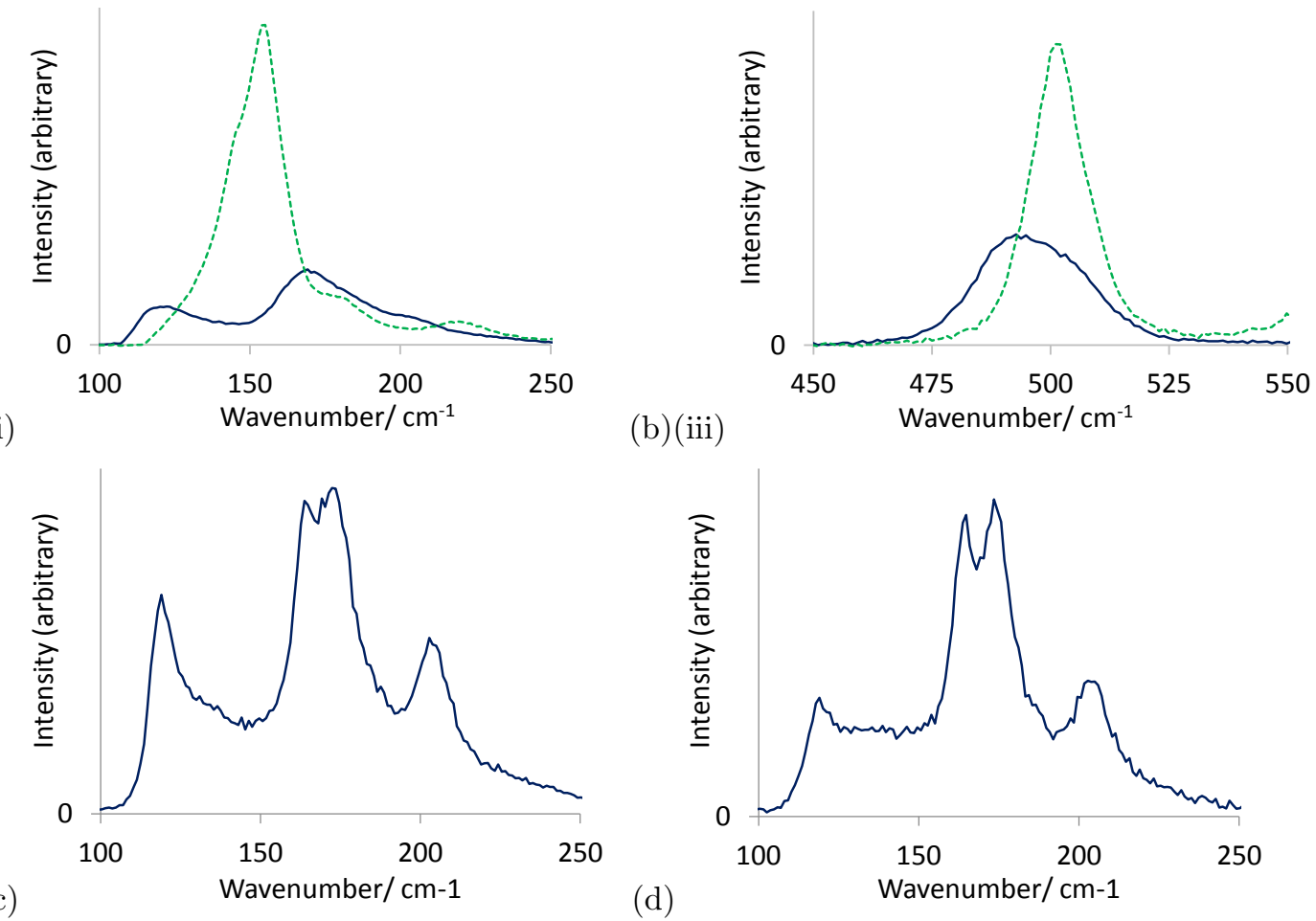

(b)(iii)

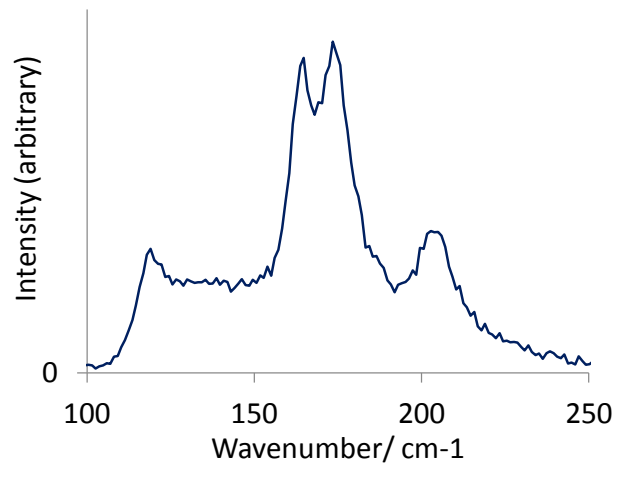

Figure S3. (a) XRD patterns of two powdered samples, one of $\alpha$ glycine and one of $\gamma$ glycine as identified with Raman spectroscopy. The glycine identified as $\alpha$ via Raman spectroscopy is shown in blue and glycine identified as $\gamma$ is shown in green. The circles represent known $\alpha$ XRD peaks while the triangles represent known $\gamma$ XRD peaks. (b)(i) Raman spectra for a typical $\alpha$ glycine crystal and a typical $\gamma$ glycine crystal. The spectra are normalised to the intensity of their highest peak at $886 \mathrm{~cm}^{-1}$ (b)(ii) The spectra of (b)(i) in the region $100-250 \mathrm{~cm}^{-1}$.(b)(ii) The spectra of (b)(i) in the region $450-550 \mathrm{~cm}$ ${ }^{-1}$. (c) and (d) Two additional $\alpha$ glycine spectra where the characteristic peaks at $164 \mathrm{~cm}^{-1}, 171 \mathrm{~cm}^{-1}$ and $203 \mathrm{~cm}^{-1}$ (which cannot be easily seen in (b)(ii)) can be more clearly seen.

The corresponding data for the $\gamma$ polymorph needle-like crystals and non-needle-like crystals are in Figure S6 As with the $\alpha$ polymorph, there is no apparent difference between the set of needle spectra and the set of non-needle Raman spectra.

\section{STATISTICS AND MODELS FOR THE NUCLEATION OF COMPETING POLYMORPHS}

As we only observe one polymorph or the other in a single well, not both, nucleation of the two polymorphs are mutually exclusive events in our system. Thus our data on the kinetics of nucleation of single crystals of competing polymorphs consist of the pair of observations, $\left(t_{n u c}, i=\alpha, \gamma\right)$, for each well where crystallisation occurred. We want to analyse this quantitative data to build the most robust model with the greatest predictive power.

The statistics of the nucleation of competing polymorphs is subtle, but fortunately, analogous data sets occur in 


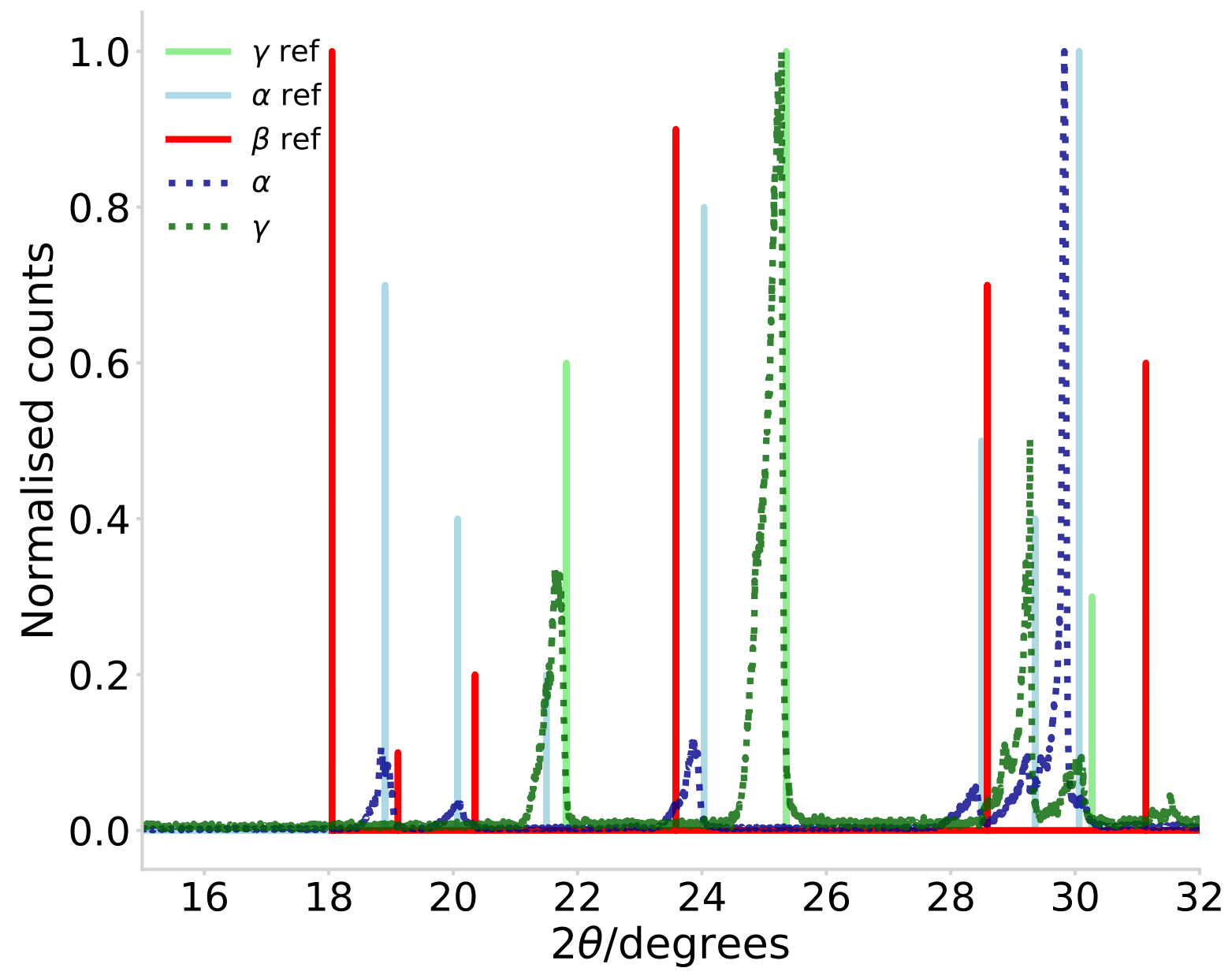

Figure S4. Experimental XRD patterns of two powdered samples, one of $\alpha$ glycine (blue dotted curve) and one of $\gamma$ glycine (green dotted curve), as identified with Raman spectroscopy. The reference peak positions for all three polymorphs are shown as solid vertical lines. Our PANalytical XPert Pro diffractometer uses JCPDS reference files, numbers 00-032-1702 $(\alpha)$, 00-002$0171(\beta)$ and 00-006-0230 $(\gamma)$. We show a $2 \theta$ range where there are three strong $\beta$ peaks that are all well separated from any $\alpha$ or $\gamma$ peak. None of the experimentally observed peaks match the $\beta$ reference file. All five patterns are normalised such that the highest peak has a height of one.

a number of other fields, in particular in medical statistics 14 18. Typically in mortality studies, there are competing illnesses or causes of death 17 19|20. An example might be a study of, say, 100 patients at risk of dying of cancer or of heart disease, where date and cause of death are recorded. As the two causes of death, like our competing polymorphs, are mutually exclusive, the data is also of the form of a pair of observations: a time, and one of a number of competing outcomes.

\section{A. Models that include only observables}

Our observables are the $I_{i}$, together with their derivatives $h_{i}$, and $P=1-I_{\alpha}-I_{\gamma}$. We can construct models using only these functions. We can create a model by specifying the two cause specific hazard (CSH) functions. 


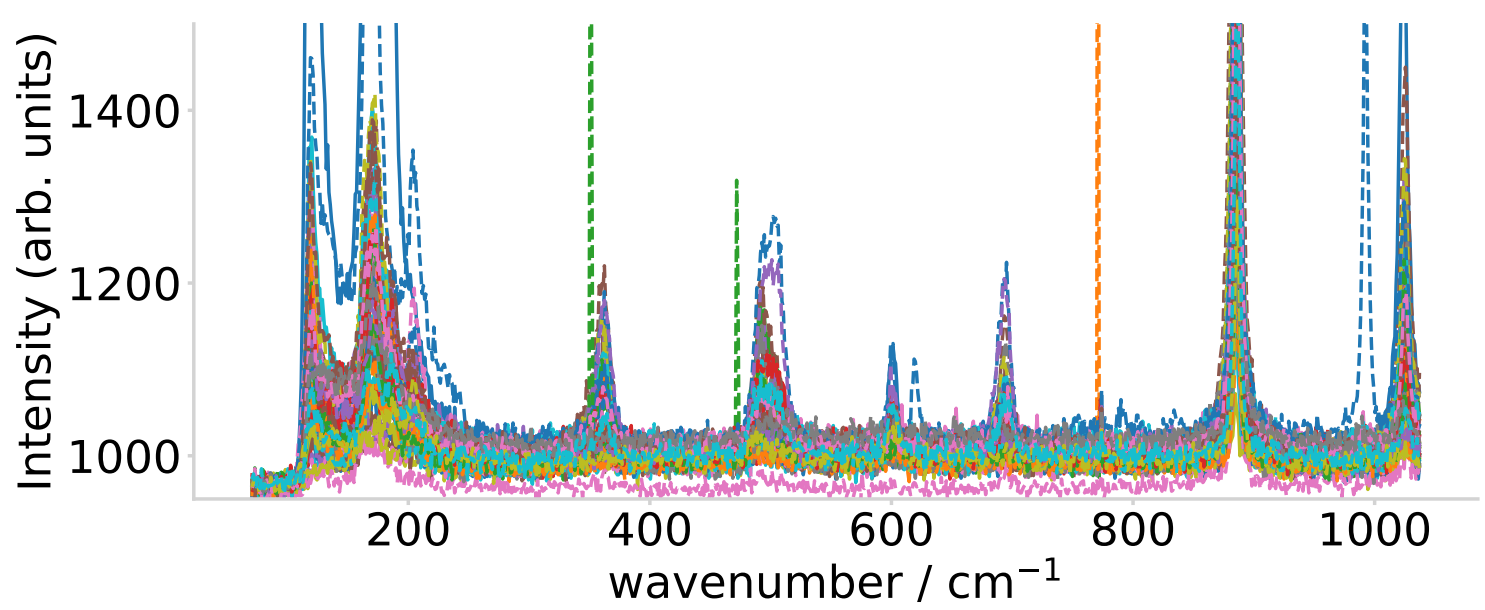

(a)

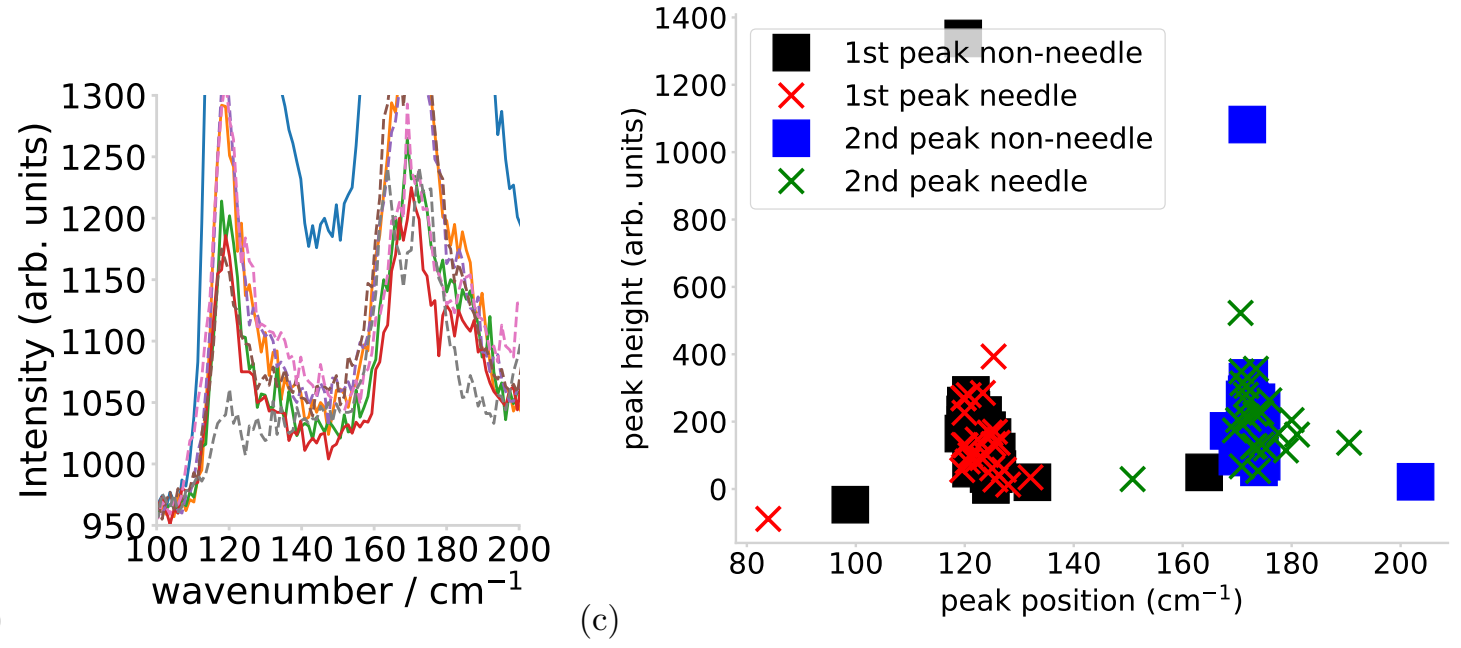

Figure S5. (a) Raman spectra for 24 non-needle like (solid curves) and 26 needle-like (dashed curves) crystals, all of which we identified as the $\alpha$ polymorph from its Raman spectrum. All crystals were obtained from run number one at a salt concentration of $250 \mathrm{mg} / \mathrm{ml}$. In two cases (green and orange dashed curves) there are anomalous spikes at single values in the data, which may be due to energetic events (cosmic rays) in the detector. (b) Four each of the non-needle (solid curves) and needle spectra (dashed curves) of (a), plotted in the range we use for fitting. (c) Plot of peak height versus peak position, obtained by a fit of two Gaussians plus a constant background to the part of the Raman spectra in (a) between wavenumbers 100 and $200 \mathrm{~cm}^{-1}$. We use two Gaussians as for $\alpha$ we expect ${ }^{9}$ one peak at around $118 \mathrm{~cm}^{-1}$, plus two peaks at 164 and $171 \mathrm{~cm}^{-1}$, where these two peaks are merged into one in the spectra of most crystals. Note that in a few instances, a peak is very weak, fitting fails, and an anomalous point, e.g., slightly negative height, is produced.

\section{Fitting procedure for models that include only observables}

The model we fit is defined by the definitions of $I_{i}$ and of $P$ :

$$
\begin{aligned}
\frac{\mathrm{d} I_{i}(t)}{\mathrm{d} t} & =P(t) h_{i}(t) \quad i=\alpha, \gamma \\
P(t) & =1-I_{\alpha}(t)-I_{\gamma}(t)
\end{aligned}
$$

with two boundary conditions $I_{i}(t=0)=0$. We can obtain an equation for $\mathrm{d} P / \mathrm{d} t$ and integrate it, to obtain

$$
P(t)=\exp \left[-\int_{0}^{t} \mathrm{~d} t^{\prime}\left(h_{\alpha}\left(t^{\prime}\right)+h_{\gamma}\left(t^{\prime}\right)\right)\right]
$$




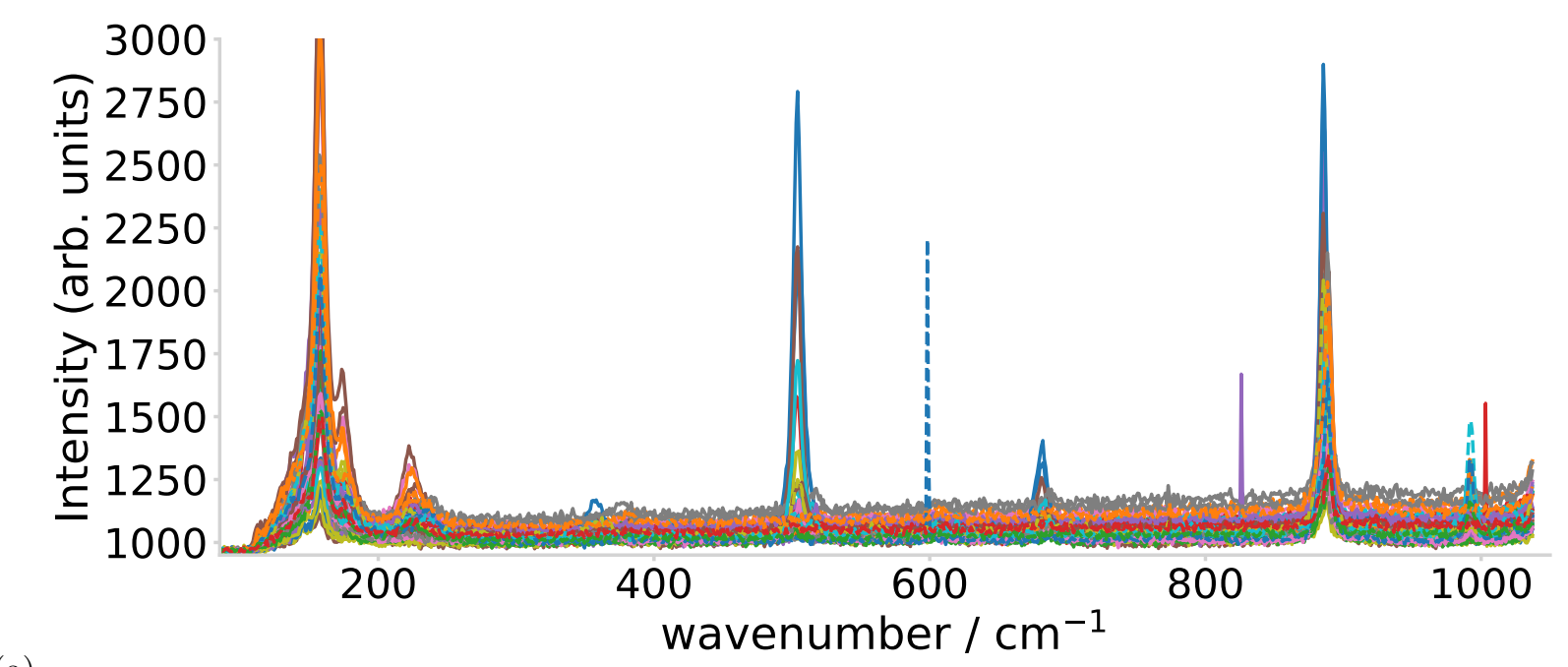

(a)

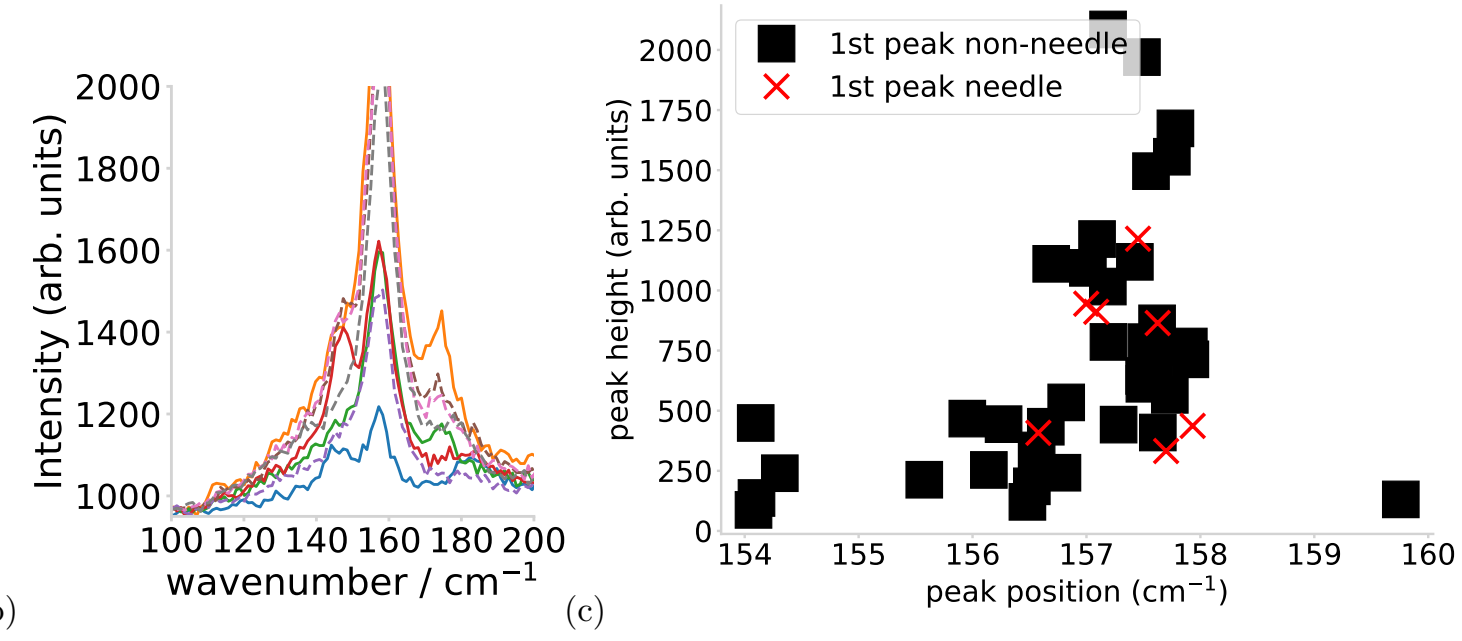

Figure S6. (a) Raman spectra for 37 non-needle like (solid curves) and 7 needle-like (dashed curves) crystals, all of which we identified as the $\gamma$ polymorph from their Raman spectra. All crystals were obtained from run number one at a salt concentration of $250 \mathrm{mg} / \mathrm{ml}$. In three cases (blue dashed curve, magenta and red solid curves) there are anomalous spikes at single values in the data, which may be due to energetic events (possibly cosmic rays) in the detector. (b) Four each of the non-needle (solid curves) and needle (dashed curves) spectra of (a), plotted in the range we use for fitting. (c) Plot of peak height versus peak position, obtained by a fit of a Gaussian plus a constant background to the part of the Raman spectra in (a) between wavenumbers 100 and $200 \mathrm{~cm}^{-1}$. We use one Gaussian as for $\gamma$ we expect ${ }^{9]}$ one peak at around $157 \mathrm{~cm}^{-1}$.

which when the $h_{i}$ are Weibull CSHs, becomes

$$
P(t)=\exp \left[-\left(t / \tau_{\alpha}\right)^{\beta_{\alpha}}-\left(t / \tau_{\gamma}\right)^{\beta_{\gamma}}\right]
$$

Equation (3) then becomes

$$
\frac{\mathrm{d} I_{i}(t)}{\mathrm{d} t}=\exp \left[-\left(t / \tau_{\alpha}\right)^{\beta_{\alpha}}-\left(t / \tau_{\gamma}\right)^{\beta_{\gamma}}\right] \beta_{i}\left(t^{\beta_{i}-1} / \tau_{i}^{\beta_{i}}\right) \quad i=\alpha, \gamma
$$

For given values of the four (two $\tau_{i}$ and two $\beta_{i}$ ) parameters, we integrate the coupled ordinary differential equations for the $I_{i}$, Eq. (7), to get the two $I_{i}(t)$ functions. To fit to data we simply vary the four parameters, and minimize the sum of the squares of the difference between the modelled $I_{i}(t)$ and observed $I_{i}(t)$. This is done using a Python $\operatorname{program} 21$. 


\section{B. Models with latent nucleation times}

Models can also be constructed that rely on latent nucleation times, i.e., on hypothetical nucleation times $t_{n u c, \alpha}$ and $t_{n u c, \gamma}$ for each well. As we only observe one polymorph for a well, for those wells where we observe nucleation we only measure the shorter one of these two times, the other one is not observable. Thus in all cases one of these times is hidden, hence the name latent time. See Beyersmann et al ${ }^{[17}$ and Geskus ${ }^{20}$ for discussion of the advantages and disadvantages of models that rely on latent times. Tsiatis ${ }^{14}$, Peterson $\frac{15}{}$, and Slud and Byar $\frac{16}{16}$ both discuss the limits of what can be inferred about $t_{n u c, \alpha}$ and $t_{n u c, \gamma}$, from data of our type. In general, the two nucleation times for a single well will be correlated, for example there may be a tendency that if one nucleation time is small in a well, that the other polymorph's nucleation time in the same well, is also small.

We can write the probability that no nucleation has occurred in a droplet at time $t$ as

$$
P(t)=\operatorname{Pr}\left(t_{n u c, \alpha}>t, t_{n u c, \gamma}>t\right)
$$

i.e., the probability that both $t_{n u c, \alpha}$ and $t_{n u c, \gamma}$ are greater than $t$.

\section{Model with independent latent nucleation times}

If the two nucleation times are independent then Eq. (8) simplifies to

$$
P(t)=\operatorname{Pr}\left(t_{n u c, \alpha}>t\right) \operatorname{Pr}\left(t_{n u c, \gamma}>t\right)
$$

If the two independent latent nucleation times are both modelled by Weibulls, then for the probability density function for $t_{n u c, i}$, we have

$$
p\left(t_{n u c, i}\right)=\beta_{i}\left(t^{\beta_{i}-1} / \tau_{i}^{\beta_{i}}\right) \exp \left[-\left(t / \tau_{i}\right)^{\beta_{i}}\right] \quad i=\alpha, \gamma
$$

and the cumulative probabilities are

$$
\operatorname{Pr}\left(t_{n u c, i}\right)=\exp \left[-\left(t / \tau_{i}\right)^{\beta_{i}}\right] \quad i=\alpha, \gamma
$$

When the latent times are independent, the rate at which $\alpha$ is observed to nucleate is just $p\left(t_{n u c, \alpha}\right)$ times the probability that nucleation of the $\gamma$ polymorph has not yet occurred, so we have for $\alpha$ nucleation

$$
\begin{aligned}
\frac{\mathrm{d} I_{\alpha}(t)}{\mathrm{d} t} & =\beta_{i}\left(t^{\beta_{\alpha}-1} / \tau_{\alpha}^{\beta_{\alpha}}\right) \exp \left[-\left(t / \tau_{\alpha}\right)^{\beta_{\alpha}}\right] \times \exp \left[-\left(t / \tau_{\gamma}\right)^{\beta_{\gamma}}\right] \\
& =\exp \left[-\left(t / \tau_{\alpha}\right)^{\beta_{\alpha}}-\left(t / \tau_{\gamma}\right)^{\beta_{\gamma}}\right] \beta_{\alpha}\left(t^{\beta_{\alpha}-1} / \tau_{\alpha}^{\beta_{\alpha}}\right)
\end{aligned}
$$

plus an analogous equation for $\mathrm{d} I_{\gamma} / \mathrm{d} t$.

These two equations are identical to Eq. (7). So our model in the previous section that modelled the observable CSHs, via Weibulls (equation (3) of the main text), can be obtained starting with latent nucleation times, and then assuming that they are both Weibull distributed, and are independent. Thus the fits of our model using only observables, are also what we would obtain from a model of independent latent times. However, it should be noted that ${ }^{14}$, although for every set of observables $I_{i}$ there is a corresponding model with independent latent times, for the same $I_{i}$ there are an infinite number of models with differently correlated latent times that yields this same $I_{i}$.

\section{Computational generation of models with independent latent nucleation times}

As we just discussed, a model with independent nucleation times is the same as our model based on observables. Nonetheless, we now briefly describe how to generate a model based on independent latent nucleation times computationally here. Then in the next sub-section we describe how we introduce correlations. To generate the model with independent times, we simulate the behaviour of a large number samples, and determine the fraction of those samples at time $t$, where nucleation has not occurred, $N(t)$. When the number of samples is sufficiently large $N(t) \approx P(t)$. For each well we need both a $t_{n u c, \alpha}$ and $t_{n u c, \gamma}$, we then select the shorter time to be the nucleation time of the well. 
For this model we need to generate two sets of variables, $t_{n u c, \alpha}$ and $t_{n u c, \gamma}$, such that both sets of variable are Weibull distributed. We can do this by generating a set of uniform random variables for each polymorph, and putting each random number through the inverse cumulative distribution function (CDF) of a Weibull distribution.

$$
y=\tau_{i}(-\ln (1-u))^{\frac{1}{\beta_{i}}} \quad i=\alpha, \gamma
$$

Where $u$ is a uniform random variable such that $0 \leq u<1$ and $y$ is the output variable which is Weibull distributed. In this way we can easily create two independent sets of Weibull distributed nucleation times. We can then vary the $\beta$ and $\tau$ values to fit our data.

\section{Model with correlated latent nucleation times}

We do not know if nucleation of the two polymorphs is correlated. So to understand the role of correlations, it is useful to have a model with variable correlations between the nucleation times of the two polymorphs. To generate the model, we start, as in the previous section, with two sets of Weibull distributed numbers for $t_{n u c, \alpha}$ and $t_{n u c, \gamma}$. In this case however we want those two sets of numbers to be correlated such that for a well with a short $\alpha$ nucleation time there is a high probability of a short $\gamma$ nucleation time

We set about generating the correlated nucleation times as follows. We generate two correlated sets of numbers, both Gaussian distributed. We then put each number into a Gaussian CDF which transforms them to uniformly distributed numbers between zero and one. After that, we put the uniformly distributed numbers into an inverse Weibull CDF, Eq. (13). At this point, we have two Weibull distributed sets of numbers that are correlated. By generating a large number of nucleation time pairs, we obtain $I_{\alpha}$ and $I_{\gamma}$ pairs where the $\alpha$ and $\gamma$ nucleation processes have the desired correlation. We used these generated distributions to fit our data, in Figure 2 of the main text.

\section{Measuring correlation}

We use Spearman's rank coefficient ${ }^{22}$ to measure correlation in this model. We briefly explain how Spearman's rank coefficient works. For each sample we have two times: $t_{n u c, \alpha}$ and $t_{n u c, \gamma}$. We rank all the $t_{n u c, \alpha}$, for all samples in order of increasing length. We do the same with the set of $t_{n u c, \gamma}$. We then measure for each pair of times the difference between the $t_{n u c, \alpha}$ rank, $r_{\alpha}$ and the $t_{n u c, \gamma} \operatorname{rank}, r_{\gamma}$. For example, if for one sample the nucleation times are $t_{n u c, \alpha}$ and $t_{n u c, \gamma}$, and if $t_{n u c, \alpha}$ is the 6th longest nucleation time $\left(r_{\alpha}=6\right)$ and $t_{n u c, \gamma}$ is the 11th longest nucleation time $\left(r_{\gamma}=11\right)$, then the difference in ranks, $r_{\gamma}-r_{\alpha}$ is 5 . Note that if $t_{n u c, \alpha}$ and $t_{n u c, \gamma}$ are perfectly correlated all their ranks will be the same.

We measure the difference in rank for each pair of variables. The sum of the square rank differences is the covariance of $r_{\alpha}$ and $r_{\gamma}$. The formula we use to calculate Spearman's rank correlation coefficient, $R_{s}$, is

$$
R_{s}=\frac{\operatorname{cov}\left(r_{\alpha}, r_{\gamma}\right)}{\sigma_{r_{\alpha}} \sigma_{r_{\gamma}}}
$$

where $\sigma_{r_{\alpha}}$ and $\sigma_{r_{\gamma}}$ are the standard deviations of all $r_{\alpha}$ and $r_{\gamma}$ respectively and $\operatorname{cov}\left(r_{\alpha}, r_{\gamma}\right)$ is the covariance of $r_{\alpha}$ and $r_{\gamma}$. When data are strongly positively correlated $R_{s}$ approaches 1 , when data are uncorrelated $R_{s}$ is close to zero and when data are strongly negatively correlated $R_{s}$ approaches -1 . The Spearman's rank correlation coefficient between the two functions in the model used in the fit in Figure 2 of the main text, is 0.95 , i.e, $\alpha$ and $\gamma$ nucleation are strongly correlated.

\section{ADDITIONAL RESULTS ON NUCLEATION}

\section{A. CIF plots for the five salt concentrations between 60 and $250 \mathrm{mg} / \mathrm{ml}$}

In Figure S7, we have plotted the CIFs: $I_{\alpha}, I_{\gamma}$ and the sum $I_{\alpha}+I_{\gamma}$, for five different salt concentrations. We do not plot results for the experiments at $300 \mathrm{mg} / \mathrm{ml} \mathrm{NaCl}$ because there is little nucleation at this salt concentration. Note the change of time scale between Figure $\mathrm{S7}(\mathrm{b})$ and (c). The experimental timescale is longer at higher $\mathrm{NaCl}$ concentrations because as salt is added, nucleation slows. The plots for $250 \mathrm{mg} / \mathrm{ml}$ are also shown in Figure 2 of the main text. The fit parameters for the Weibull fits are in Table S2. 


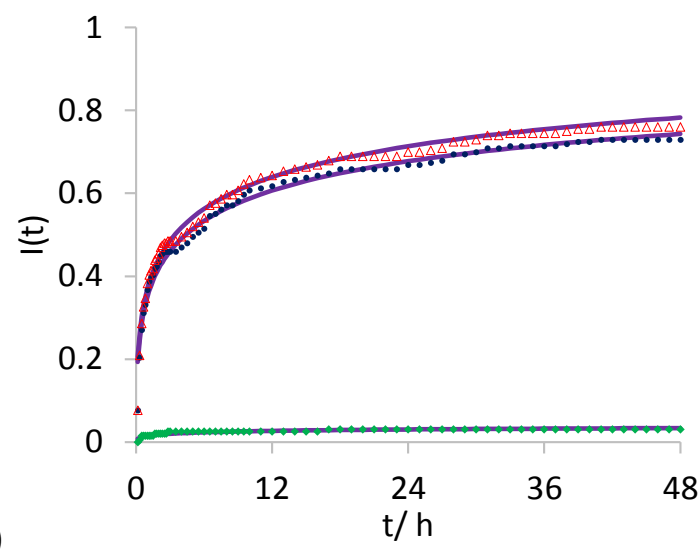

(a)

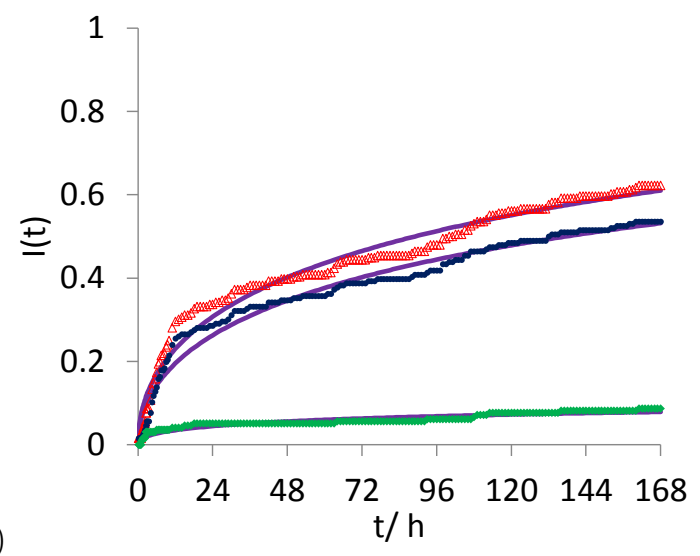

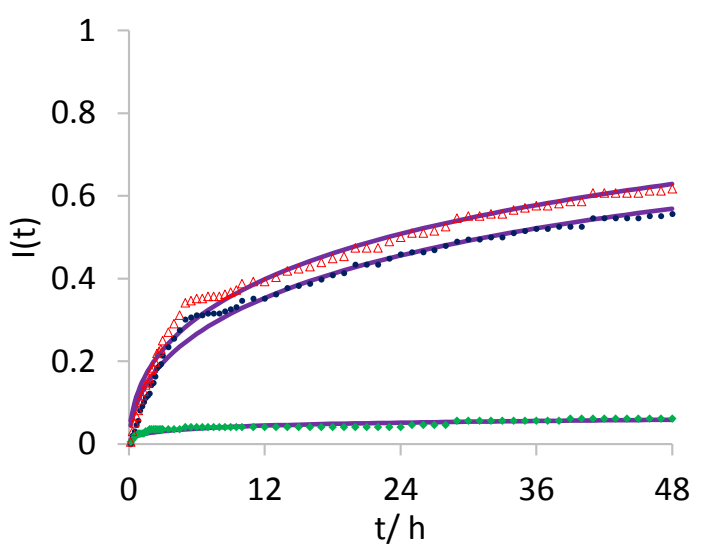

(b)

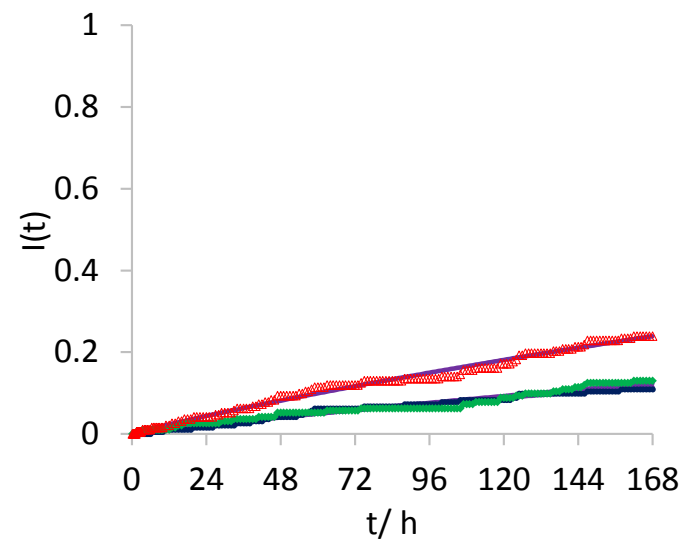

(e)

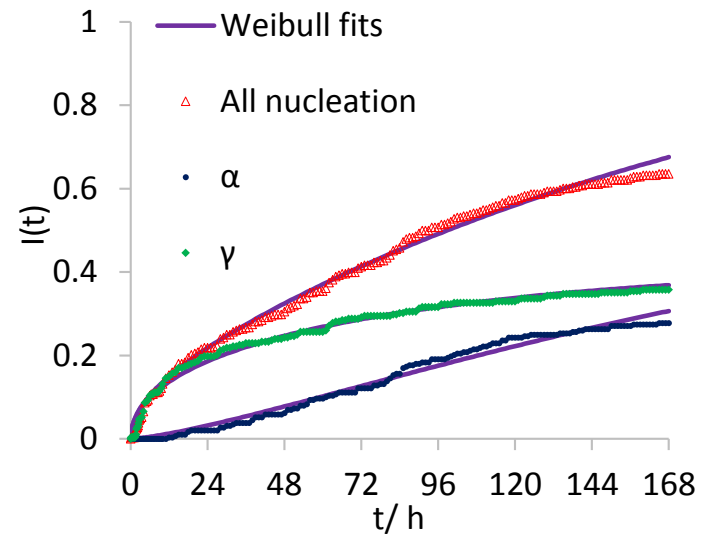

Figure S7. Plots of CIFs for five salt concentrations: (a) $60 \mathrm{mg} / \mathrm{ml}$, (b) $90 \mathrm{mg} / \mathrm{ml}$, (c) $150 \mathrm{mg} / \mathrm{ml}$, (d) $200 \mathrm{mg} / \mathrm{ml}$, and (e) 250 $\mathrm{mg} / \mathrm{ml}$. The legend in (e) applies to all plots. The points are our data: $I_{\alpha}$ (blue), $I_{\gamma}$ (green) and $I_{\alpha}+I_{\gamma}$ (red), respectively. Purple curves are fits of models with Weibull CSHs to the data.

\section{B. Increasing salt concentration favours the $\gamma$ polymorph}

We plot the final fraction of crystals that are in the $\gamma$ polymorph, in Figure S8. This is for experiments were carried out at $\mathrm{NaCl}$ concentrations $c_{\mathrm{NaCl}}$ from $60 \mathrm{mg} / \mathrm{ml}$ to $300 \mathrm{mg} / \mathrm{ml}$. At least two runs were carried out at each salt concentration. We define a run as the set of nucleation times recorded from one 96 -well microplate. On completing each run, the fraction of wells containing each polymorph was determined.

In Figure S8 the variation of the final fraction of crystals in the $\gamma$ polymorph, $f_{\gamma}$, is modelled using a logistic function. The fit is shown as a black curve in Figure S8. The logistic function is

$$
f_{\gamma}\left(c_{N a C l}\right)=\frac{1}{1+\exp \left[-\left(c_{N a C l}-c_{1 / 2}\right) / c_{S W}\right]}
$$


Table S2. Best-fit values for fits of models with Weibull CSHs to the CIFS for $\alpha$ and $\gamma$ nucleation.

\begin{tabular}{c|cccc|cc}
$\begin{array}{c}\mathrm{NaCl} \\
(\mathrm{mg} / \mathrm{ml})\end{array}$ & $\tau_{\alpha}(\mathrm{h})$ & $\beta_{\alpha}$ & $\tau_{\gamma}(\mathrm{h})$ & $\beta_{\gamma}$ & $R_{\alpha}^{2}$ & $R_{\gamma}^{2}$ \\
\hline 60 & 7.49 & 0.26 & $4.74 \times 10^{5}$ & 0.28 & 1.00 & 1.00 \\
90 & 57.2 & 0.49 & $5.84 \times 10^{5}$ & 0.26 & 0.99 & 1.00 \\
150 & 245 & 0.49 & $3.35 \times 10^{4}$ & 0.40 & 0.99 & 1.00 \\
200 & 1650 & 0.89 & 1250 & 0.97 & 1.00 & 1.00 \\
250 & 244 & 1.41 & 610 & 0.48 & 0.99 & 0.99 \\
\hline
\end{tabular}

$R_{i}^{2}, i=\alpha, \gamma$, is the $R^{2}$ value for comparison of the fit $I_{i}$ to the data.

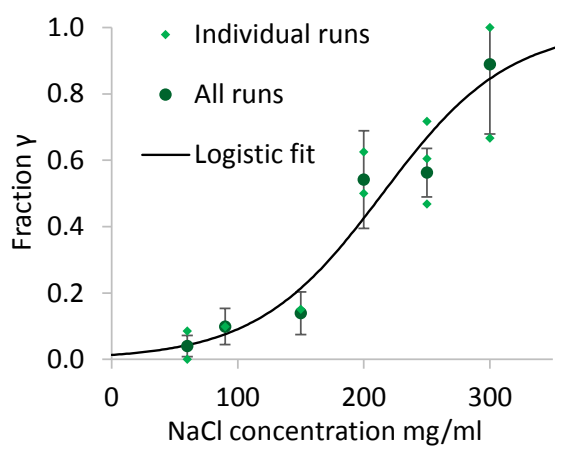

Figure S8. The final fraction of wells that contain the $\gamma$ polymorph, $f_{\gamma}$, plotted as a function of NaCl concentration. The dark green circles are the overall fraction averaged over all runs at a single $\mathrm{NaCl}$ concentration. The smaller green diamonds are the fractions in individual runs. The solid curve is a fit of a logistic function to $f_{\gamma}=n_{\gamma} /\left[n_{\alpha}+n_{\gamma}\right]$, where $n_{\alpha}$ and $n_{\gamma}$ are the number of wells containing $\alpha$ crystals and $\gamma$ crystals, respectively. The error bars shown have a total height of $2\left[f_{\gamma}\left(1-f_{\gamma}\right) /\left(n_{\alpha}+n_{\gamma}\right)\right]^{1 / 2}$, where $n_{\alpha}$ is the number of wells containing $\alpha$ crystals and $n_{\gamma}$ is the number of wells containing $\gamma$ crystals.

and it has two parameters: $c_{1 / 2}$ and $c_{S W}$. As we can see in Figure $\mathrm{S} 8$, this functional form fits our data well. The best-fit parameters are $c_{1 / 2}=215 \mathrm{mg} / \mathrm{ml}$, and $c_{S W}=100 \mathrm{mg} / \mathrm{ml}$. The parameter, $c_{1 / 2}$, is an estimate for the salt concentration at which half the samples are $\alpha$ and half $\gamma$. There is broad region, a few hundred $\mathrm{mg} / \mathrm{ml}$, over which we go from a region with very small, but non-zero, amounts of the $\gamma$, to a large majority of the crystals in the $\gamma$ polymorph. The $c_{S W}$ parameter can be used as an estimator of the width of this region. The data is in Table S3.

Table S3. Values of $f_{\gamma}$ at six $\mathrm{NaCl}$ concentrations.

\begin{tabular}{|c|ccc|}
\hline $\mathrm{NaCl}(\mathrm{mg} / \mathrm{ml})$ & $f_{\gamma}$ & $n_{\alpha}$ & $n_{\gamma}$ \\
\hline 60 & 0.04 & 143 & 6 \\
90 & 0.10 & 109 & 12 \\
150 & 0.14 & 102 & 17 \\
200 & 0.54 & 21 & 25 \\
250 & 0.56 & 80 & 103 \\
300 & 0.89 & 1 & 8 \\
\hline
\end{tabular}

$f_{\gamma}$ is the fraction of wells at the end of the experiment, where the the crystal(s) are of the $\gamma$ polymorph. Also shown are the total numbers of wells with $\alpha$ and with $\gamma$ crystals, $n_{\alpha}$ and $n_{\gamma}$, respectively. 


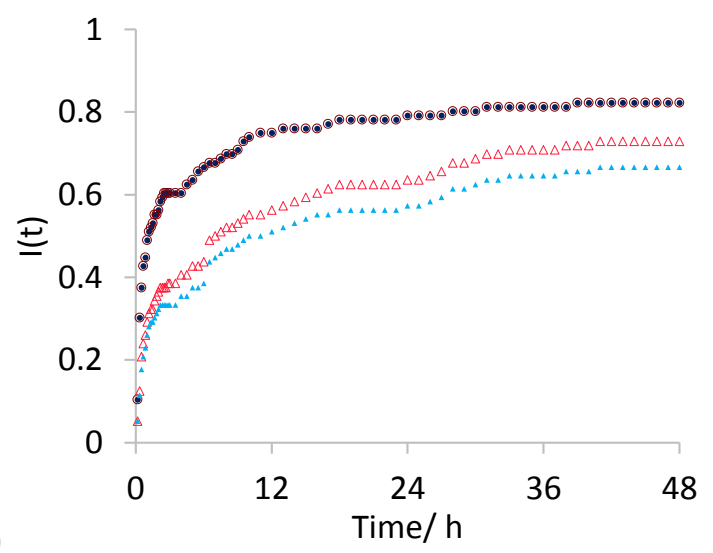

(a)

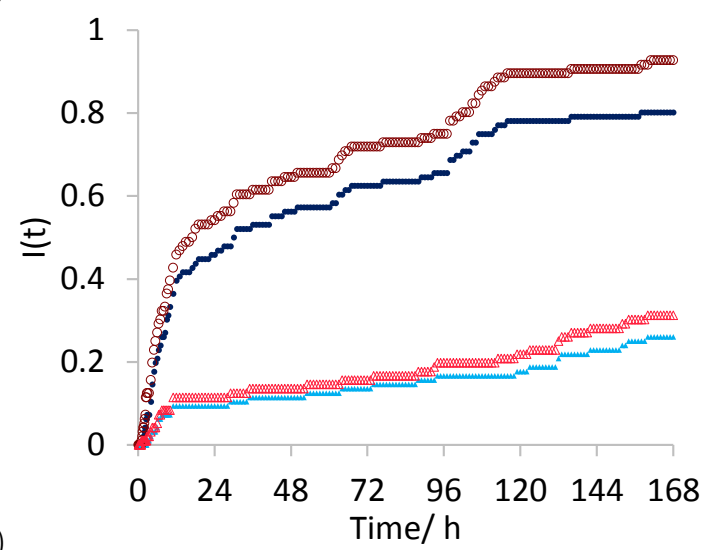

(b)

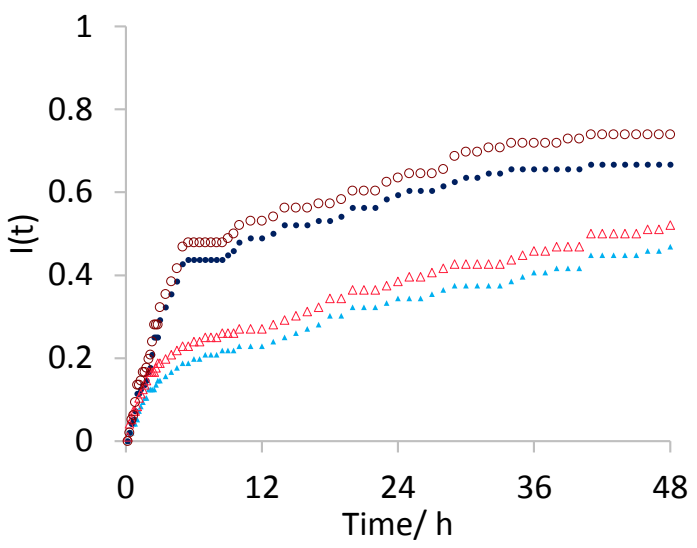

(c)

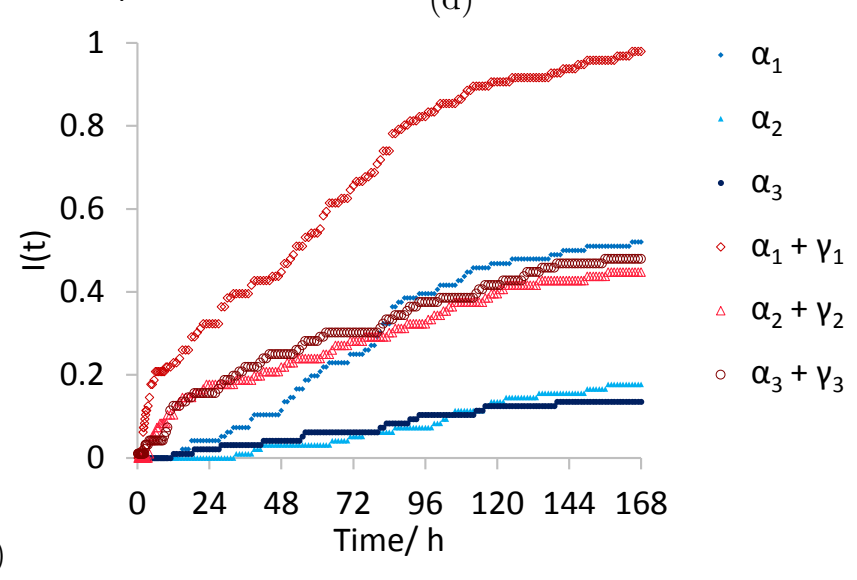

Figure S9. CIFs for individual runs at $\mathrm{NaCl}$ concentrations: (a) $60 \mathrm{mg} / \mathrm{ml}$, (b) $90 \mathrm{mg} / \mathrm{ml}$, (c) $150 \mathrm{mg} / \mathrm{ml}$, (d) $200 \mathrm{mg} / \mathrm{ml}$ and (e) $250 \mathrm{mg} / \mathrm{ml}$. The key in (e) applies to all 5 plots. There are 2 runs in (a) to (d), and 3 runs in (e). For each run we plot $I_{\alpha}$ as closed blue symbols, and $I_{\alpha}+I_{\gamma}$ as open red/brown symbols. For example, in (a) for the first run no $\gamma$ crystals form, so the solid and open symbols are on top of each other as $I_{\gamma}=0$, while for the second run a small number of $\gamma$ crystals start to form after a few hours, and so the pale blue closed and open red symbols move apart.

\section{Rates of nucleation, and total amount of nucleation are not well reproducible, relative rates and fractions are reproducible}

The nucleation time cumulative incidence functions (CIFs) are plotted for each individual run in Figure S9 The CIFs in Figure S7 were obtained by combining the data in these individual runs. The number of wells in which nucleation occurs, varies from run to run, i.e., reproducibility of the amount of nucleation is poor. What is reproducible, is the fraction of nucleation events of each polymorph. In Figure S9 $\gamma$ nucleation corresponds to the difference between the all-nucleation CIFs and the $\alpha$ nucleation CIFs for each run. We see at low $\mathrm{NaCl}$ concentrations nucleation is dominated by the $\alpha$ polymorph and this phenomenon is reproducible between runs. Even when two runs have a 
significantly different amount of nucleation occurring, the relative amount of the nucleation of each polymorph is similar. For example at $150 \mathrm{mg} / \mathrm{ml} \mathrm{NaCl}$, see Figure S9(c), one run (dark red) has about three times more nucleation events than the other (light red). However, for both runs, about $15 \%$ of the total nucleation events give the $\gamma$ polymorph.

It should also be noted that the distribution of nucleation times is reproducibly different for the two polymorphs at the high salt concentration of $250 \mathrm{mg} / \mathrm{ml} \mathrm{NaCl}$, see Figure S9. The $\gamma$ nucleation time distribution initially has a very fast relative nucleation rate which slows down over time in all of the runs, while the $\alpha$ nucleation time distribution initially has a very slow relative nucleation rate which speeds up over time giving an 's-shaped' curve. This is well illustrated by the median nucleation times of the subpopulations of $\alpha$ nucleating wells and $\gamma$ nucleating wells for each run. The median $\alpha$ nucleation times for the three runs are $77 \mathrm{~h}, 102 \mathrm{~h}$ and $81 \mathrm{~h}$, while median $\gamma$ nucleation times for those runs are $13.5 \mathrm{~h}, 14 \mathrm{~h}$ and $34 \mathrm{~h}$ respectively. We can therefore say, in addition to the fraction of nucleation that corresponds to each polymorph being reproducible, the relative change in the effective nucleation rates of the two polymorphs is also reproducible.
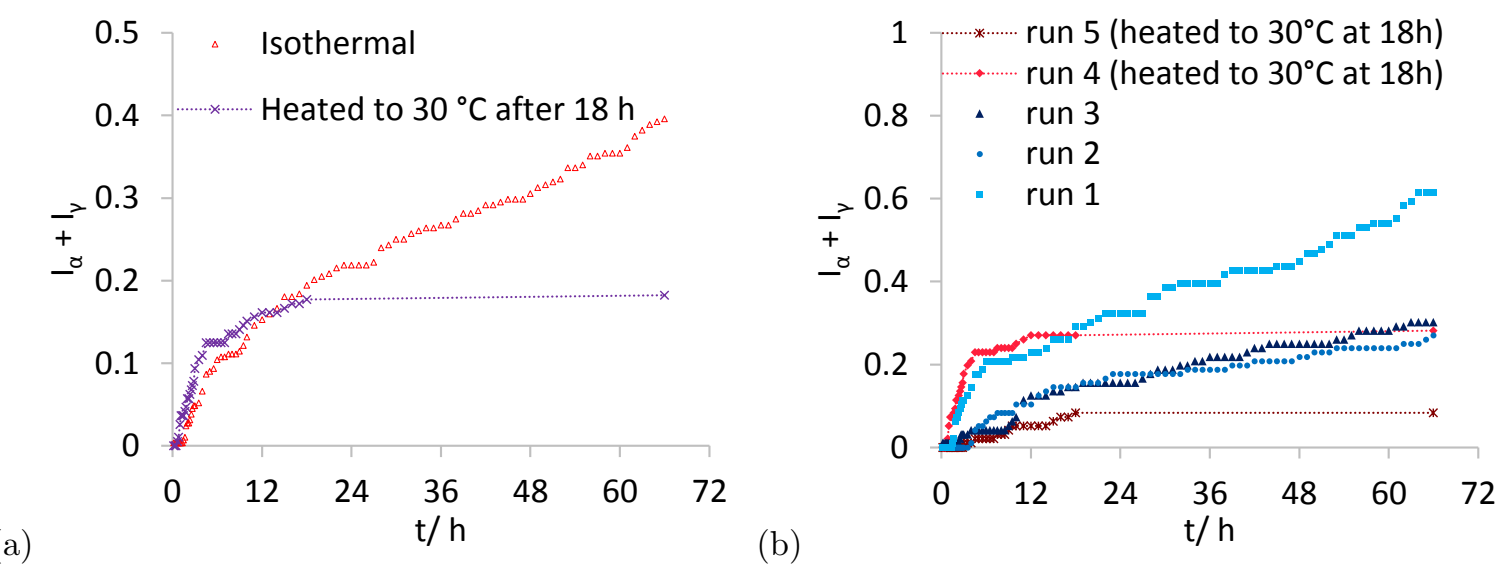

Figure S10. Plots of the sum of the two CIF functions, $I_{\alpha}+I_{\gamma}$, i.e., the fraction of wells where crystallisation has occurred, is plotted as a function of time. This is at $250 \mathrm{mg} / \mathrm{ml}$ salt. (a) The red triangles are the average $I_{\alpha}+I_{\gamma}$, for all three isothermal runs, and the purple crosses are is the average of two runs that are at $21^{\circ} \mathrm{C}$ for the first $18 \mathrm{~h}$, after which the microplate is maintained at $30.4{ }^{\circ} \mathrm{C}$ for the following $48 \mathrm{~h}$. (b) Here we have plotted the individual runs of the systems that were averaged to obtain the data in (a). This is two runs heated to $30^{\circ} \mathrm{C}$ after $18 \mathrm{~h}$ (runs 4 and 5 ), and the isothermal individual runs (runs $1,2$ and 3$)$.

\section{Time-dependent supersaturation increases polymorph purity}

In Figure $\mathrm{S} 10$ (a), we have plotted the sum of the two CIFs, $I_{\alpha}+I_{\gamma}$ for both isothermal experiments, and experiments where the temperature is increased from room temperature (close to $21^{\circ} \mathrm{C} 10$ ) to $30.4{ }^{\circ} \mathrm{C}$, after 18 hours. All runs are at $250 \mathrm{mg} / \mathrm{ml} \mathrm{NaCl}$. Note that for the experiments warmed to $30.4{ }^{\circ} \mathrm{C}$, nucleation is almost completely stopped. In Figure S10(b), we show the individual runs that make up Figure S10(a). We can see the trends observed in the individual runs are the same as we observe in the datasets where all runs under the same conditions are combined.

\section{E. Individual purity vs times}

We have looked at how polymorph composition varies over time. Here we show that the runs that make up the datasets plotted in Figure 4 follow the same trends. The runs are plotted in Figure S11, and data is shown in Table S4. 


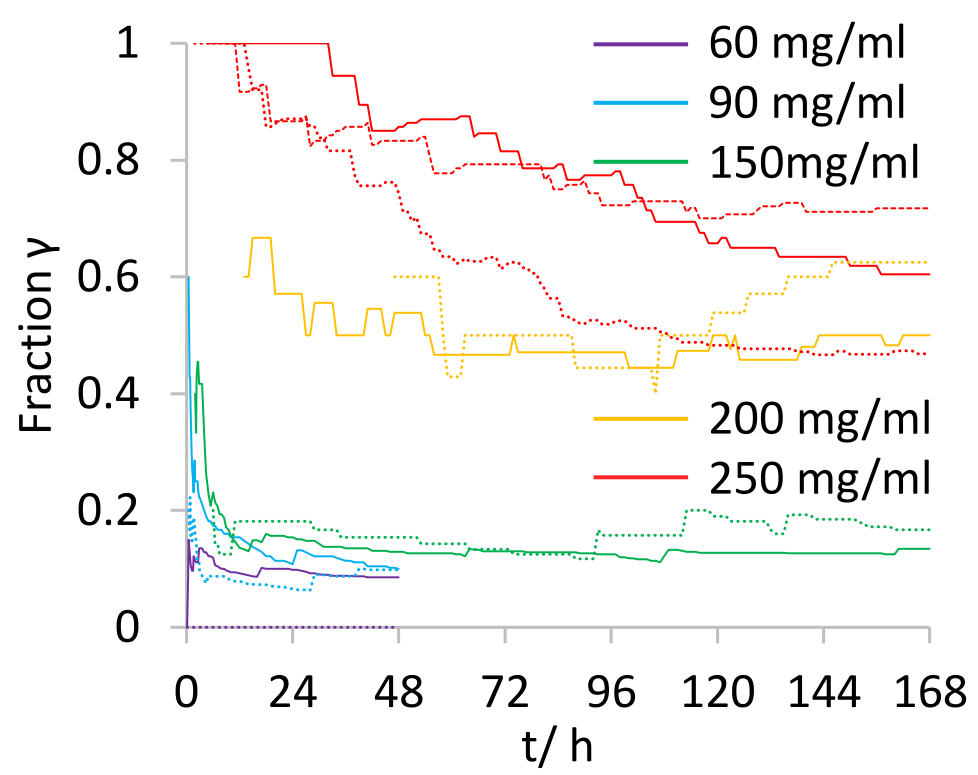

Figure S11. Polymorph composition as a function of time. The composition is the fraction of the wells where crystallisation has occured, that contain the $\gamma$ polymorph. The lines start at the point when five nucleation events have occurred. Each curve is one run, and the colour indicates the salt concentration. Different runs at the same concentration are distinguished by being solid, dashed and dotted.

Table S4. Results of individual runs, for the fraction of $\gamma$ at early times, and at the end.

\begin{tabular}{|c|cc|cc|}
\hline $\mathrm{NaCl}$ conc $/ \mathrm{mg} / \mathrm{ml}$ & Fraction $\gamma$ at $n_{\geq 10}$ & Final $\gamma$ fraction & $n_{\geq 10}$ & $t_{n \geq 10} / \mathrm{h}$ \\
\hline 60 & 0.0 & 0.0 & 10 & 0.16 \\
60 & 0.083 & 0.086 & 12 & 0.33 \\
\hline 90 & 0.30 & 0.10 & 10 & 1.16 \\
90 & 0.15 & 0.099 & 13 & 1.0 \\
\hline 150 & 0.45 & 0.13 & 11 & 2.5 \\
150 & 0.18 & 0.17 & 11 & 11 \\
\hline 200 & 0.50 & 0.50 & 10 & 34 \\
200 & 0.40 & 0.63 & 10 & 106 \\
\hline 250 & 1.0 & 0.47 & 10 & 2.66 \\
250 & 1.0 & 0.60 & 10 & 10 \\
250 & 1.0 & 0.72 & 11 & 11 \\
\hline
\end{tabular}

The second column is the fraction of crystals in the $\gamma$ polymorph, at a time, $t_{n \geq 10}$, early in the experiment. $t_{n \geq 10}$ is

the earliest observation time at which we have 10 or more nucleation events; the precise number of nucleation events, $n_{\geq 10}$, is in column four. The third column is the fraction of the $\gamma$ polymorph at the end of the experiment.

\section{ADDITIONAL RESULTS ON GROWTH RATES AND CRYSTAL HABITS}

\section{A. The effect of growth rates on the error in nucleation time measurements}

Our measurements for nucleation times are only accurate if the time for nucleation, i.e., for the crystal to cross the nucleation barrier and start growing irreversibly, is much larger than the time taken for the crystal to grow from just past the barrier, to a size large enough to be visible.

We have plotted the sizes of $10 \alpha$ and $10 \gamma$ crystals, at $90 \mathrm{mg} / \mathrm{ml} \mathrm{NaCl}$ in Figure S12. Data for $250 \mathrm{mg} / \mathrm{ml} \mathrm{NaCl}$ are in Figure 5 of the main text. Note that growth rates vary with salt concentration. The crystals grow faster at 90 $\mathrm{mg} / \mathrm{ml}$ of salt than $250 \mathrm{mg} / \mathrm{ml}$. We focus on the $\gamma$ crystals for estimating nucleaction time errors as their growth is 


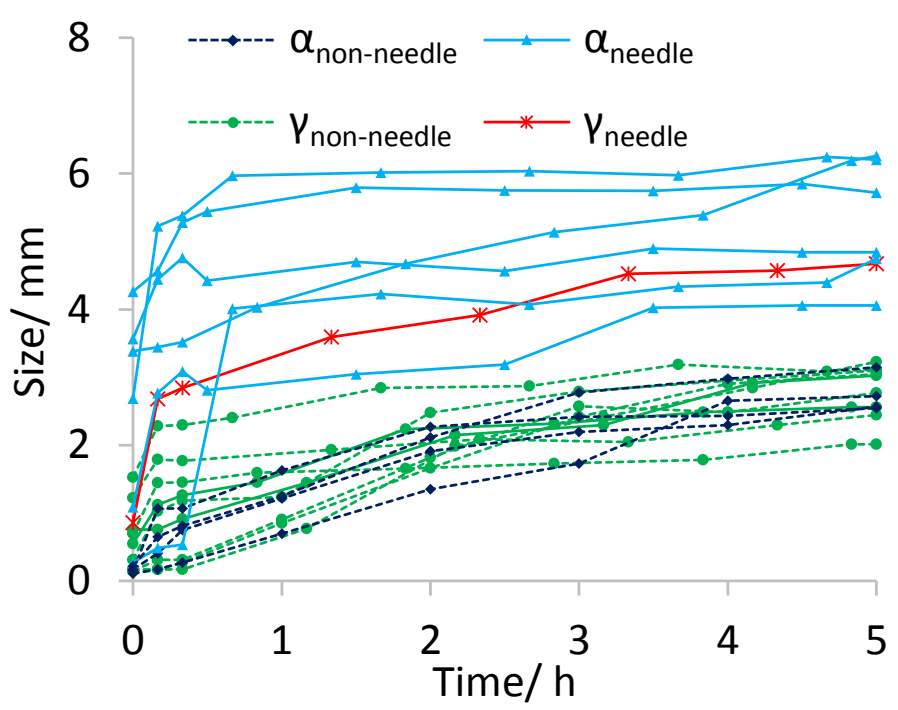

Figure S12. Plot of the size of a crystal, as a function of time. The $\mathrm{NaCl}$ concentrations is $90 \mathrm{mg} / \mathrm{ml}$. Data is shown for $10 \alpha$ and $10 \gamma$ crystals. The sizes of $\alpha$ crystals are shown as light blue (needles) and black (non-needles) lines-and-points. The sizes of $\gamma$ crystals are shown as red (needles) and green (non-needles) lines-and-points. For each crystal $t=0$ is defined as the time of the first image in which there is a visible crystal.

slightly slower than that of the $\alpha$ crystals. Here the growth rate for the $\gamma$ crystals is around $2 \mathrm{~mm} / \mathrm{h}$. This means the error in our nucleation time measurements should be less than 10 minutes in most cases. This is a small error for all but the very shortest nucleation times.

For a salt concentration of $250 \mathrm{mg} / \mathrm{ml}$, we plotted the sizes of $10 \alpha$ and $10 \gamma$ crystals, as a function of time, in Figure 5 of the main text. Most but not all of the $\gamma$ crystals are growing at rate of around $0.2 \mathrm{~mm} / \mathrm{h}$. This implies that our measured nucleation times are on average just over 30 mins too long, i.e., we first see a $\gamma$ crystal about 30 mins after it nucleated as a microscopic nucleus. A few $\alpha$ crystals are also growing at around $0.2 \mathrm{~mm} / \mathrm{h}$, but most are initially growing of order 10 times faster. Our runs at $250 \mathrm{mg} / \mathrm{ml}$ salt are of $168 \mathrm{~h}$ duration, so except for nucleation at early times, a 30 mins error is a relatively small error.

Table S5. Mean glycine sizes at initial detection and at two subsequent times, for both $\alpha$ and $\gamma$ polymorphs.

\begin{tabular}{|c|c|cc|cc|}
\hline \multirow{2}{*}{$\mathrm{NaCl}$ conc / mg/ml } & Time / mins & \multicolumn{4}{|c|}{ Size/ mm } \\
& & \multicolumn{2}{|c|}{ mean } & \multicolumn{2}{|c|}{ Interquartile range } \\
& & $\alpha$ & $\gamma$ & $\alpha$ & $\gamma$ \\
\hline 90 & 0 & $1.6 \pm 0.5$ & $0.6 \pm 0.1$ & 3.2 & 0.69 \\
& 20 & $2.5 \pm 0.7$ & $1.2 \pm 0.3$ & 4.0 & 1.5 \\
& 300 & $4.3 \pm 0.5$ & $3.0 \pm 0.2$ & 3.0 & 0.56 \\
\hline 250 & 0 & $0.5 \pm 0.1$ & $0.17 \pm 0.02$ & 0.74 & 0.11 \\
& 20 & $0.9 \pm 0.2$ & $0.23 \pm 0.01$ & 1.2 & 0.025 \\
& 300 & $1.8 \pm 0.3$ & $0.9 \pm 0.1$ & 2.3 & 0.23 \\
\hline
\end{tabular}

We estimate the uncertainties of the mean values with error estimates that are the standard deviation of the measured sizes, divided by the square root of the number of crystals measured (10 crystals of each polymorph at each concentration). We measure the width of the distribution of sizes of the crystals via the interquartile range.

\section{B. Variation in growth rates between crystals}

In Table S5, we present crystal sizes at three times, from the same data set as used in Figure 5 and Figure S12. In this table, the interquartile range (IQR) is $Q_{3}-Q_{1}$, where $Q_{1}$ is the first quartile of the distribution of crystal sizes, 
i.e., the size at which $25 \%$ of the crystals are smaller and $75 \%$ are larger, and $Q_{3}$ is the size where $75 \%$ are smaller and $25 \%$ are larger. The IQR is a convenient measure of the spread in crystal sizes as it is relatively insensitive to outliers (unlike the standard deviation), and we have outliers in the crystal size, see Figure S12. It is clear that the growth rates of both polymorphs vary widely between one crystal and another. This is especially true for the $\alpha$ polymorph where at early times the IQR is larger than the mean.
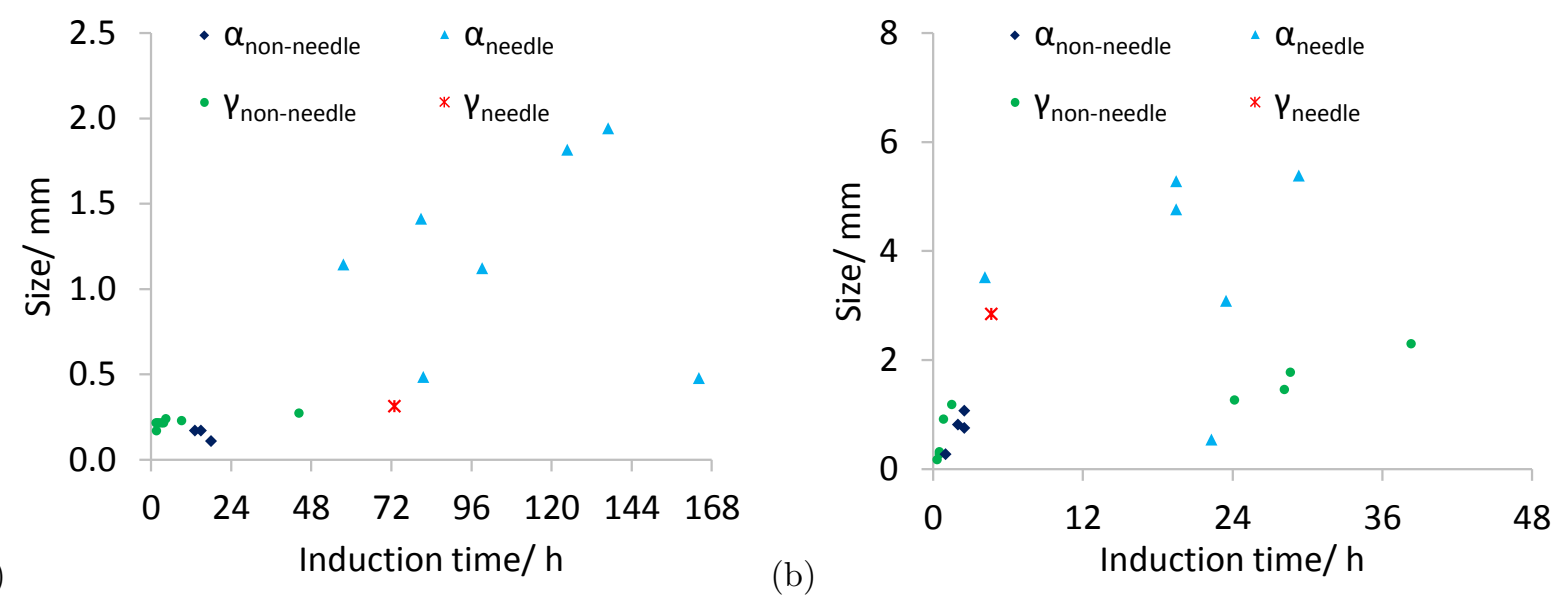

Figure S13. Here we plot the size of crystals 20 mins after they were initially detected, as a function of the time at which they nucleated. In (a) the salt concentration is $250 \mathrm{mg} / \mathrm{ml}$, and in (b) it is $90 \mathrm{mg} / \mathrm{ml}$.

\section{Growth rate and nucleation time}

In the main text we showed that needle-like crystals tend to have faster growth rates. We have also showed that crystals that nucleate at later times are more likely to be needle-like. It follows that the crystals with longer nucleation times generally have faster growth rates. As we have discussed, the growth rate of crystals is difficult to quantify for our data because the growth rate changes with time. Here we plot the size of a crystal 20 mins after it is initially detected against nucleation time. This is shown in Figure S13. We see that at both salt concentrations, the earliest nucleating crystals are small at 20 mins.

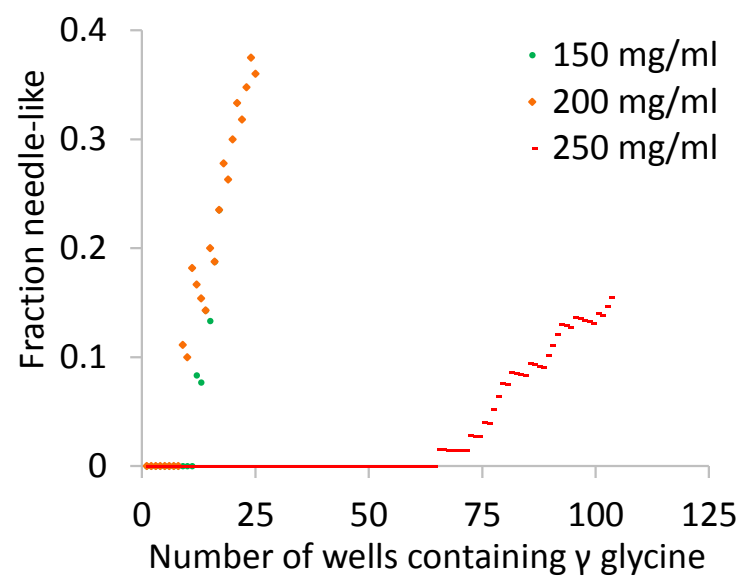

Figure S14. For the $\gamma$ polymorph, we plot the fraction of crystals with a needle-like habit, as a function of the total number of wells that contain crystals of that polymorph. 


\section{Nucleation time and crystal habit}

For $\gamma$ crystals at high salt concentrations, we observe the same correlation between crystal habit and nucleation time, as we did for the $\alpha$ polymorph (Figure 7). The data for the $\gamma$ polymorph are shown in Figure S14. We see the same trend as for the $\alpha$ polymorph, with non-needle-like crystals nucleating at early times, and needle-like crystals at late times, although the fraction of needles is lower for the $\gamma$ polymorph. At low salt concentrations, there article too few $\gamma$ crystals to make clear statements. For example, at $60 \mathrm{mg} / \mathrm{ml}$ and $90 \mathrm{mg} / \mathrm{ml}$ there are only six and twelve $\gamma$ nucleation events in total, respectively.

${ }^{1}$ L. Li and N. Rodrguez-Hornedo, J. Cryst. Growth 121, 33 (1992)

${ }^{2}$ G. Han, P. S. Chow, and R. B. H. Tan, Crys. Growth Design 12, 2213 (2012).

${ }^{3}$ G. Han, P. S. Chow, and R. B. H. Tan, Cryst. Growth Design 15, 1082 (2015).

${ }^{4}$ R. Dowling, R. J. Davey, R. A. Curtis, G. Han, S. K. Poornachary, P. S. Chow, and R. B. H. Tan, Chem. Commun. 46, 5924 (2010).

${ }^{5}$ M. Sultana and K. F. Jensen, Cryst. Growth Design 12, 6260 (2012).

${ }^{6}$ A. I. Toldy, A. Z. M. Badruddoza, L. Zheng, T. A. Hatton, R. Gunawan, R. Rajagopalan, and S. A. Khan, Crys. Growth Des. 12, 3977 (2012).

${ }^{7}$ X. Yang, X. Wang, and C. B. Ching, J. Chem. Eng. Data 53, 1133 (2008).

${ }^{8}$ Y. Yi, D. Hatziavramidis, A. S. Myerson, M. Waldo, V. G. Beylin, and J. Mustakis, Ind. Eng. Chem. Res. 44, 5427 (2005).

${ }^{9}$ Y. Shi and L. Wang, J. Phys. D App. Phys. 38, 3741 (2005)

${ }^{10}$ L. J. Little, R. P. Sear, and J. L. Keddie, Crys. Growth Design 15, 5345 (2015).

${ }^{11}$ I. Weissbuch, V. Y. Torbeev, L. Leiserowitz, and M. Lahav, Ang. Chem. Int. Ed. 44, 3226 (2005).

${ }^{12}$ E. Seyedhosseini, M. Ivanov, V. Bystrov, I. Bdikin, P. Zelenovskiy, V. Y. Shur, A. Kudryavtsev, E. D. Mishina, A. S. Sigov, and A. L. Kholkin, Cryst. Growth Design 14, 2831 (2014).

${ }^{13}$ D. Isakov, D. Petukhova, S. Vasilev, A. Nuraeva, T. Khazamov, E. Seyedhosseini, P. Zelenovskiy, V. Y. Shur, and A. L. Kholkin, Cryst. Growth Design 14, 4138 (2014)

${ }^{14}$ A. Tsiatis, Proc. Nat. Acad. Sci. 72, 20 (1975).

${ }^{15}$ A. V. Peterson, Proc. Nat. Acad. Sci. 73, 11 (1976).

${ }^{16}$ E. Slud and D. Byar, Biometrics 44, 265 (1988).

${ }^{17}$ J. Beyersmann, A. Latouche, A. Buchhol, and M. Schumacher, Statist. Med. 28, 956 (2009).

${ }^{18}$ P. K. Andersen, R. B. Geskus, T. de Witte, and H. Putter, Int. J. Epidemiology 41, 861 (2012)

${ }^{19}$ J. J. Dignam, Q. Zhang, and M. N. Korcherginsky, Clin Cancer Res , 2301 (2012).

${ }^{20}$ R. B. Geskus, Data Analysis with Competing Risks and Intermediate States (Chapman and Hall / CRC, 2015).

${ }^{21} \mathrm{~L} . \mathrm{J}$. Little, A tortoise and the hare story: The relationship between induction time and polymorphism in glycine crystallisation (PhD thesis, University of Surrey, 2017).

${ }^{22}$ J. I. Hoffman, in Biostatistics for Medical and Biomedical Practitioners edited by J. I. Hoffman (Academic Press, 2015$)$ pp. 513 - 536. 